\title{
Oxidative Modifications of Parkin Underlie its Selective Neuroprotection in Adult Human Brain
}

Jacqueline M. Tokarew ${ }^{1,2}$ *, Daniel N. El-Kodsi ${ }^{1,2} *$, Nathalie A. Lengacher ${ }^{1}$, Travis K. Fehr ${ }^{1,2}$, Angela P. Nguyen ${ }^{1}$, Brian O’Nuallain ${ }^{3}$, Ming Jin ${ }^{3}$, Jasmine M. Khan ${ }^{1}$, Andy C. H. Ng ${ }^{1}$, Juan Li ${ }^{1}$, Qiubo Jiang ${ }^{1}$, Mei Zhang ${ }^{4}$, Liqun Wang ${ }^{3}$, Rajib Sengupta ${ }^{5,5^{*}}$, Kathryn R. Barber ${ }^{6}$, An Tran ${ }^{6}$, Stephanie Zandee ${ }^{7}$, Xiajun Dong ${ }^{8}$, Clemens R. Scherzer ${ }^{8}$, Alexandre Prat ${ }^{7}$, Eve Tsai ${ }^{1,9}$, Masashi Takanashi ${ }^{10}$, Nobutaka Hattori ${ }^{10}$, Jennifer A. Chan ${ }^{11}$, Andrew B. West ${ }^{12}$, Arne Holmgren ${ }^{5, \%}$, Lawrence Puente $^{13}$, Gary S. Shaw ${ }^{6}$, Gergely Toth ${ }^{14}$, John M. Woulfe ${ }^{1,4}$, Peggy Taylor ${ }^{3}$, Julianna J. Tomlinson ${ }^{1,15}$ \# and Michael G. Schlossmacher ${ }^{1,15,16}$ \#

${ }^{1}$ Program in Neuroscience, Ottawa Hospital Research Institute, Ottawa, ON, Canada

${ }^{2}$ Graduate Program in Cellular and Molecular Medicine (Neuroscience), Faculty of Medicine, University of Ottawa, Ottawa, ON, Canada

${ }^{3}$ BioLegend Inc., Dedham, MA., USA

${ }^{4}$ Department of Pathology and Laboratory Medicine, The Ottawa Hospital, Ottawa, ON, Canada

${ }^{5}$ Department of Biochemistry, Karolinska Institute, Stockholm, Sweden;

${ }^{5 *}$ Present Address: Amity Institute of Biotechnology, Amity University, Kolkata, West Bengal 700135, India

${ }^{6}$ Department of Biochemistry, University of Western Ontario, London, ON, Canada

${ }^{7}$ Department of Neuroscience, Faculty of Medicine, University of Montreal, Montreal, QC, Canada

${ }^{8}$ Ann Romney Center for Neurologic Diseases, Brigham \& Women's Hospital, Boston, MA, USA

${ }^{9}$ Division of Neurosurgery, Department of Medicine, The Ottawa Hospital, Ottawa, ON, Canada

${ }^{10}$ Department of Neurology, Juntendo University School of Medicine, Tokyo, Japan

${ }^{11}$ Department of Pathology \& Laboratory Medicine, University of Calgary, Calgary, AB, Canada

${ }^{12}$ Departments of Neurobiology and Pharmacology \& Cancer Biology, Duke University, Durham, NC, USA

${ }^{13}$ Proteomics Core Facility, Ottawa Hospital Research Institute, Ottawa, ON, Canada

${ }^{14}$ Institute of Organic Chemistry, Research Center for Natural Sciences, Budapest, Hungary

${ }^{15}$ University of Ottawa Brain and Mind Research Institute, Ottawa, ON, Canada

${ }^{16}$ Division of Neurology, Department of Medicine, The Ottawa Hospital, Ottawa, ON, Canada

*these authors contributed equally; ${ }^{\%}$ deceased 
bioRxiv preprint doi: https://doi.org/10.1101/2020.02.19.953034; this version posted February 20, 2020. The copyright holder for this preprint (which was not certified by peer review) is the author/funder. All rights reserved. No reuse allowed without permission.

\#Lead contacts: jtomlins@uottawa.ca; mschlossmacher@ohri.ca 


\section{SUMMARY}

The mechanisms by which Parkinson disease-linked parkin confers neuroprotection of human dopamine cells remain elusive. We hypothesized that its cysteines mediate multiple anti-oxidant effects in the midbrain. By studying $>60$ control specimens, we found that in adult human brain but not in skeletal muscle- parkin is mostly aggregated and insoluble due to oxidative modifications, such as at $\mathrm{C} 253$. In vitro, parkin's oxidation directly reduces hydrogen peroxide $\left(\mathrm{H}_{2} \mathrm{O}_{2}\right)$ to water. In parkin-deficient human brain, $\mathrm{H}_{2} \mathrm{O}_{2}$ concentrations are elevated. In dopamine toxicity studies, wild-type parkin -but not disease-associated mutants- prevents neural death by lowering $\mathrm{H}_{2} \mathrm{O}_{2}$ and sequestering radicals within insoluble aggregates. Parkin conjugates dopamine metabolites at the human-specific residue C95 and augments melanin formation in vitro. Using epitope-mapped antibodies, we found that in adult Substantia nigra neurons parkin localizes to neuromelanin within LAMP-3/CD63-positive lysosomes. We conclude that parkin's own oxidation, previously considered a loss-of-function event, underlies three neuroprotective effects in adult midbrain: its cysteines participate in $\mathrm{H}_{2} \mathrm{O}_{2}$ reduction, dopamine radical conjugation and the formation of neuromelanin. 


\section{INTRODUCTION}

Parkinson disease (PD) remains an incurable disorder of the human brain. Bi-allelic mutations in the PRKN gene, which encodes parkin, lead to young-onset, autosomal-recessive PD (ARPD) ${ }^{1}$. Clinicopathological studies of parkin-deficient brains have demonstrated restricted cell loss in the $S$. nigra and L. coeruleus, two nuclei synthesizing dopamine (DA). The field has not yet explained the selective neurodegeneration of PRKN-linked ARPD vs. other forms of PD.

Parkin, a principally cytosolic protein, has been associated with diverse cellular functions, foremost related to ubiquitin ligase activity and mitochondrial integrity ${ }^{2}$. However, none of these roles has explained its neuroprotective selectivity, and animal models of genomic prkn deletion have failed to reproduce DA cell loss and parkinsonism. This observation could be due to compensatory mechanisms, a shorter life span, or the uniqueness of human DA metabolism. The latter is exemplified by the generation of neuromelanin in dopaminergic cells beginning in late childhood ${ }^{3,4}$. Nevertheless, select prkn-null models have revealed changes in high energy-producing cells of flies ${ }^{5}$ and murine brain indicative of augmented oxidative stress ${ }^{6-}$ 8. We postulated that wild-type parkin promotes redox homeostasis in vivo.

Redox equilibrium frequently involves cysteine-based chemistry; there, thiols are subject to oxidative modifications by reactive oxygen-, reactive nitrogen- and reactive electrophilic species (ROS, RNS, RES) ${ }^{9}$, some of which are reversible. Proteins irreversibly conjugated by RES, including by select DA radicals, are degraded, secreted or sequestered within inclusions. It is thought that this process underlies neuromelanin formation ${ }^{10}$.

Human parkin contains 35 cysteines $^{1}$. Several reports previously demonstrated its sensitivity to ROS and RES in cells ${ }^{11,12}$. RNS also attack parkin, and NO-/ $\mathrm{NO}_{2}$-modified variants have been described in cells and tissue isolates ${ }^{13}$. Such oxidative modifications, which reduce parkin's ubiquitin ligase activity ex vivo, have been previously interpreted as a 'loss-offunction' ${ }^{12,14-16}$ in vivo. In contrast, we hypothesized that parkin's oxidation ensures redox homeostasis by actively neutralizing ROS, RNS and DA radicals in human brain, and that in parkin's absence their accumulation leads to selective neurodegeneration in PRKN-linked ARPD. Hence, we explored parkin's metabolism in adult human midbrain and pons $v s$. cortex, spinal cord, skeletal muscle, tested its contribution to redox equilibrium, and studied parkin modifications during DA metabolism.

\section{RESULTS}

\section{Parkin is mostly insoluble in the ageing human midbrain}

Parkin's metabolism in the human nervous system has remained largely unexplored ${ }^{17}$. We serially fractionated $>40$ cortices (age range, 5-85 years) and 20 midbrain specimens (26-82 years) from individuals who had died from a non-neurological cause (Fig. 1, Extended Data Fig. 1; Supplementary Table 1). We found that before age 20 years, nearly $50 \%$ of cortical parkin was found in soluble fractions generated by salt [Tris-NaCl]- and mild detergent [Triton X-100]-containing buffers (Fig. 1a,b; Extended Data Fig.1a). In contrast, after age 50 years, parkin was found nearly exclusively $(>90 \%)$ in the $2 \%$ SDS-soluble fraction and $30 \%$ SDS extract of the final pellet in brainstem and forebrain specimens, which included the S.nigra, L.coeruleus, striatum and cortex (Fig. 1a-c). The requirement for SDS to solubilize parkin suggested it had been aggregated, which is consistent with its co-distribution with LC3B, a 
phagosomal marker of aggregated proteins (Fig. 1d). In soluble brain fractions from older humans, we did not detect any truncated species of parkin using several, specific antibodies. Despite the protein's solubility loss, $P R K N$ mRNA was detectable in individually examined nigral and cortical neurons from all age groups (Fig. 1e). Moreover, transcript levels were independent of age (Extended Data Fig. 1d).

Using a logistic regression model with parkin as the dependent variable, we found that its solubility in human brain correlated with age (Fig. 1f), where the age coefficient was -0.0601 (95\% CI: -0.106 to $-0.024 ; \mathrm{P}=0.004)$. When using age to classify soluble $v s$. insoluble, the area under the receiver operating characteristic (ROC) curve was 0.8 . This transition to insolubility occurred between the age range of 28 (low sensitivity; high specificity) and 42 years (high sensitivity; low specificity). We confirmed that parkin's solubility change was not caused by tissue freezing, was independent upon post mortem interval (PMI) and sex, and it was not affected by the $\mathrm{pH}$ of extraction buffers (Extended Data Fig. 2; not shown).

The age-dependent partitioning of parkin was not seen for any other protein examined, which included PD-linked proteins, e.g., DJ-1 and $\alpha$-synuclein (Fig. 1a,g) and organelleassociated markers, e.g.: cytosolic glyoxalase-1, peroxiredoxin-1 and -3; endoplasmic reticulumassociated calnexin; and mitochondrial VDAC and MnSOD (Fig. 1h; Extended Data Fig. 1b,c; and data not shown). Interestingly, its partitioning was organ- and species-specific. In contrast to human brain, parkin remained soluble in skeletal muscle and spinal cord specimens from older individuals (Fig. 1i; Extended Data Fig. 1e). In the brains of aged mice (at 22 months), rats (14 months) and cynomolgus monkey (5 years), parkin remained soluble even when the length and conditions of PMIs were matched to those of human tissues (Extended Data Fig. 1f; data not shown). We concluded that parkin's solubility loss is relatively unique to human brain ageing.

\section{Parkin is oxidized in adult human brain}

When human brain fractions were examined under non-reducing conditions, we detected parkin proteins ranging in $M_{r}$ from $>52$ to $270 \mathrm{kDa}$, invariably in the form of smears (Fig. 1j). These species were abundant in SDS extracts and reduced to parkin's monomer $(\sim 52.5 \mathrm{kDa})$ by adding dithiotreitol (DTT) or $\beta$-mercaptoethanol ( $\beta$-ME). This is shown, for example, in specimens from both normal and neurologically diseased cases (Fig. 1 j,k). No such reactivity was seen in SDS-extracts of PRKN-linked ARPD brains, demonstrating specificity. Examples of oxidative modifications are shown in Extended Data Fig. 3. Under the same conditions, high $M_{r}$ protein variants were rarely seen for other PD proteins, e.g., DJ-1 and $\alpha$-synuclein. The latter showed oligomers only in select brains from older subjects (not shown). Of note, the formation of high $M_{r}$ parkin was not due to oxidation in vitro, as brain specimens were fractionated in the presence of iodoacetamide (IAA) to protect thiols; they also did not arise from ubiquitin adducts, which cannot be removed by reducing agents.

These findings demonstrated that a pool of parkin had undergone oxidation in vivo. Indeed, using logistic regression analyses, we found that in the same brain specimens $\mathrm{H}_{2} \mathrm{O}_{2}$ concentrations significantly correlated with age (Fig. 11). Moreover, parkin's solubility loss paralleled the rise in $\mathrm{H}_{2} \mathrm{O}_{2}$; the latter's coefficient was -0.939 (95\% CI: -2.256 to -0.248 ; $\mathrm{P}=0.0415$; Fig. 1m).

\section{Redox stressors directly modify parkin and alter its solubility}

To investigate mechanisms by which its solubility is altered by redox stress, we exposed recombinant (r-), tag-less, human parkin to oxidants in vitro. The exposure of highly purified r- 
parkin to rising concentrations of $\mathrm{H}_{2} \mathrm{O}_{2}, \mathrm{DA}$, the DA metabolite aminochrome, and mitochondrial toxins, e.g., CCCP and rotenone, generated high $M_{r}$ smears and progressive insolubility (Fig. 2a; Extended Data Fig. 4a-c)). Some of these biochemical changes were reversible, as seen for insoluble, $\mathrm{H}_{2} \mathrm{O}_{2}$-oxidized r-parkin upon exposure to DTT (Fig. 2b). Others could not be reduced, such as when irreversible, covalent modifications had occurred (Extended Data Fig. 4a-c; data not shown). Blocking access to parkin's thiols prevented its oxidation (see below). Demonstrating sensitivity to bi-directional redox changes, exposure of native r-parkin to excess DTT also rendered it insoluble (Extended Data Figs. 3,4d).

\section{Redox stress alters parkin's structure}

The progressive insolubility of parkin with increasing oxidative stress in vitro and in vivo suggested that the nascent protein responds to redox alterations with structural change(s). We tested this by dynamic light scattering (DLS) and far-UV-circular dichroism (CD). When we monitored r-parkin during spontaneous oxidization using DLS (Extended Data Fig. 4g), we observed a shift in r-parkin's hydrodynamic diameter from $5.1 \mathrm{~nm}$, representing a folded monomer at the start, to multiple peaks with larger diameters $5 \mathrm{hrs}$ later; the latter indicated multimer formation. These oxidation-induced changes were reversible by DTT.

To further monitor structural changes, we analyzed the effects of spontaneous oxidation in naïve and $\mathrm{H}_{2} \mathrm{O}_{2}$-treated r-parkin by $\mathrm{CD}$ (Fig. 2c,d; Extended Data Fig. 4e,f). At the start, soluble fractions contained both $\alpha$-helically ordered as well as unstructured proteins (Fig. 2b;

Extended Data Fig. 4e). After five days, r-parkin preparations were centrifuged, and soluble and insoluble fractions re-analyzed (Fig. 2d; Extended Data Fig. 4f). There, we found a marked shift by CD from an $\alpha$-helically ordered (and unstructured) content to increased amounts of $\beta$ pleated sheet-positive r-parkin in insoluble, aggregate-rich fractions. This shift was more pronounced in $\mathrm{H}_{2} \mathrm{O}_{2}$-oxidized protein preparations (Extended Data Fig. 4f).

\section{Hydrogen peroxide modifies parkin at multiple cysteines}

To further confirm that oxidation of parkin cysteines led to redox state change and insolubility, we used liquid chromatography-based mass spectrometry (LC-MS/MS). We employed a serial IAA-DTT-NEM fingerprinting approach to differentiate reduced thiols (tagged by IAA) from reversibly oxidized thiols (reduced by DTT and then tagged by N-ethylmaleimide (NEM)). We first asked whether oxidation of r-parkin led to changes in thiol redox state regardless of solubility. At higher $\mathrm{H}_{2} \mathrm{O}_{2}$ concentrations, 32 of 35 cysteines $(91.4 \%)$ were identified; $>93.8 \%$ were found modified at least once. Using Scaffold PTM-software, we recorded the anticipated rise in NEM-tagged cysteines following $\mathrm{H}_{2} \mathrm{O}_{2}$ treatment (Extended Data Fig. 4h). Oxidative modifications were identified at parkin's RING domains and at non-RING-based cysteines. Total cysteine modifications were quantified by MaxQuant software ${ }^{18}$. As shown in Fig. 2e, there was a significant loss of IAA signals for r-parkin's cysteines in $\mathrm{H}_{2} \mathrm{O}_{2}$-treated samples $(\mathrm{P}=0.0016)$, reflecting a decrease in reduced thiols.

In turn, when comparing cysteine oxidation in soluble $v s$. insoluble fractions of both untreated and oxidized r-parkin, IAA signals (i.e., free thiols) were significantly reduced in the pellets $(\mathrm{P}<0.0001$; Fig. 2f). Modifications at other residues, e.g., methionines, did not correlate with r-parkin insolubility (not shown). In these studies, residue C253 (RING1 domain) was frequently detected as oxidized. These results established that $\mathrm{H}_{2} \mathrm{O}_{2}$-induced oxidation of cysteine-based thiols conferred r-parkin's insolubility. 
We next examined whether these thiol oxidations also occur in human brain-derived parkin by LC-MS/MS. Here, we mapped cysteines in reduced and oxidized states for the 52.5 $\mathrm{kDa}$ monomer purified from cortex specimens of neurological controls. The latter included irreversible oxidation events, such as to sulfonic acid at C253 (Fig. 2g-i) and C95 (Fig. 2j,k), as well as reversible ones, e.g., at C253, detected in insoluble parkin (see Extended Data Table 1).

\section{Parkin reduces hydrogen peroxide to water}

Redox reactions transfer oxygen, electrons and $\mathrm{H}^{+}$between the reactants. We therefore asked whether parkin's oxidation led to $\mathrm{H}_{2} \mathrm{O}_{2}$ reduction (Fig. 3; Extended Data Fig. 5a-f). Indeed, we found that r-parkin directly lowered $\mathrm{H}_{2} \mathrm{O}_{2}$ levels in a time- and concentration-dependent manner (Fig. 3a; Extended Data Fig. 5c). This reaction (e.g., 2R-SH+ $\mathrm{H}_{2} \mathrm{O}_{2} \rightarrow \mathrm{R}-\mathrm{S}-\mathrm{S}-\mathrm{R}+2 \mathrm{H}_{2} \mathrm{O}$ ) was dependent on thiol integrity, because pre-treatment with IAA abolished it (Fig. 3b). Parkin's reducing capacity was confirmed in parallel studies employing a different $\mathrm{H}_{2} \mathrm{O}_{2}$ assay \& .

We next examined the reducing capacities of other proteins, e.g., r-DJ-1, a C-terminal RING2 peptide (r-parkin $321 \mathrm{C}$ ), and two ARPD-linked mutants, p.G328E and p.C431F (Fig. 3c-d, Extended Data Fig. 5d-f). There, r-DJ-1 and r-parkin $321 \mathrm{C}$ showed negligible $\mathrm{H}_{2} \mathrm{O}_{2}$-reduction, and the point mutants conferred less activity than wild-type r-parkin (Fig. 3c; Extended Data Fig. 5d,e). These reducing effects correlated with r-parkin's own oxidation, as revealed by SDSPAGE immediately after $\mathrm{H}_{2} \mathrm{O}_{2}$ exposure (Fig. 3d). We speculated that this activity by r-parkin was dependent on reactive thiol content. We tested this using the Ellman's reagent. There, wildtype r-parkin, r-parkin $321 \mathrm{C}$ and r-DJ-1 showed the predicted number of reactive thiols, whereas the point mutants p.C431F and p.G328E had fewer accessible thiols (Fig. 3e). As predicted, we recorded a linear correlation between thiol molar equivalencies and $\mathrm{H}_{2} \mathrm{O}_{2}$-reducing capacities (Fig. 3f).

\section{Hydrogen peroxide is elevated in parkin-deficient brain}

To determine whether parkin oxidation conferred ROS reduction in adult brain, we quantified $\mathrm{H}_{2} \mathrm{O}_{2}$ concentration in cortices from human ARPD subjects and age-, PMI-, ethnicity- and brain region-matched control specimens $(n=4 \text { each })^{1,19}$. There, we found a significant elevation in $\mathrm{H}_{2} \mathrm{O}_{2}$ concentrations in parkin-deficient brain lysates $(\mathrm{P}<0.05$; Fig. 3g). Of note, in specimens of non- $P R K N$-linked cases with parkinsonism, $\mathrm{H}_{2} \mathrm{O}_{2}$ levels were similar to those of age-matched controls (Fig. 11). We concluded that parkin contributes to redox homeostasis in human brain. In related mouse experiments, we identified that parkin's regulation of neural $\mathrm{H}_{2} \mathrm{O}_{2}$ occurs in the cytosol $^{\&}$.

\section{Parkin prevents dopamine toxicity by lowering hydrogen peroxide}

To explore its selective neuroprotection, we revisited the role of parkin in DA-induced toxicity ${ }^{13}$. We first tested its redox-related functions in human neuroblastoma M17 cells. Here, wild-type parkin, but not ARPD mutants p.G328E and p.C341F, protected cells from rising DA levels (Fig. 4a; Extended Data Fig. 6a). Moreover, DA-exposure resulted in a significant rise in $\mathrm{H}_{2} \mathrm{O}_{2}$ concentrations, which wild-type -but not mutant- parkin reversed (Fig. 4b). These ex vivo results validated our in vitro and in vivo findings (Fig. 3). In stable M17 cells, protection from DA toxicity paralleled $P R K N$ expression levels (Fig. 4c); approximately 4 ng neutralized each $\mu \mathrm{M}$ of

\footnotetext{
${ }^{\&}$ El-Kodsi D et al., Parkinson Disease-Linked Parkin Lowers Cytosolic Oxidative Stress in Feedback Loop with Glutathione (2020), manuscript in preparation
} 
DA. Neuroprotection also correlated with parkin's own oxidation and progressive insolubility. Importantly, these modifications were not reducible by DTT (Fig. 4d,e), indicating irreversible thiol changes, which we reasoned were due to covalent DA adduct formation ${ }^{13}$.

\section{Parkin binds dopamine radicals at cysteine 95}

We asked whether parkin directly aided in the neutralization of DA through conjugation at specific residues ${ }^{14}$. Indeed, full-length r-parkin bound DA and aminochrome adducts, as detected for example by nitro-blue-tetrazolium staining after aminochrome exposure (Fig. 4f; and data not shown). Using LC-MS/MS to map these modifications, we identified several aminochrome, DA and indole-5,6-quinone adduct-carrying peptides specific to treated r-parkin (Extended Data Fig. 6c,d). Intriguingly, the most frequently modified residue was C95, a primate sequencespecific cysteine (Fig. 4g; Extended Data Fig. 6c).

\section{Parkin augments melanin formation}

Given the dynamic associations between r-parkin, DA exposure, RES conjugation and aggregate formation, we tested whether these influenced melanin formation in vitro. Polymerization of DA into melanin occurs in a spontaneous, oxidation-dependent manner via intermediates, such as indole-5,6-quinone, and is blocked by glutathione ${ }^{10,20}$ Wild-type parkin and two point mutants, p.C431F and p.G328E, all augmented melanin formation in a time- and concentration-dependent manner (Fig. 4h; Extended Data Fig. 6b). We next sought to validate a role for parkin in melanin formation in vivo.

\section{Parkin colocalizes with neuromelanin in adult $\boldsymbol{S}$. nigra neurons}

To visualize parkin's subcellular localization in adult brain, we carried out routine microscopy studies using newly developed, monoclonal antibodies to human parkin; these had been epitopemapped and validated regarding their specificity (e.g., Extended Data Fig. 7). When staining serial sections of midbrains from control individuals, three antibodies revealed intense, granular parkin staining in the cytoplasm of pigmented neurons from adults (age $\geq 55$ years), albeit at varying intensity levels amongst them (clones-D, -E and -G; Extended Data Fig.8a). We counted anti-parkin reactivity in $>83 \%$ of anti-tyrosine hydroxylase-positive cells and in many non-pigmented neurons of the midbrain and cortex (Extended Data Fig. 8b,c).

By immunohistochemistry, in S. nigra neurons anti-parkin signals heavily overlapped with neuromelanin pigment (Extended Data Fig 8d). Sections from individuals with parkinsonism (not linked to PRKN mutations) showed the same anti-parkin reactivities in surviving neurons and overlapping with extracellular pigment following neuronal degeneration. We found weaker anti-parkin reactivity when examining $S$. nigra sections of younger subjects ( $\leq 33$ years), which matched their paucity in neuromelanin content (Extended Data Fig. 8d,e).

\section{Parkin localizes to LAMP-3 ${ }^{+}$-lysosomes within nigral neurons}

In screening organelle markers by immunohistochemistry, we detected highly similar staining between antibodies to parkin and lysosome-associated protein-3 (LAMP-3/CD63) reactivities (Extended Data Fig. 8f). Antibodies to LC3B -but no mitochondrial antigens (e.g., MnSOD, VDAC)- generated much less signal overlap (not shown). Because neuromelanin granules are thought to arise within non-degradative lysosomes ${ }^{21}$, we carried out immunofluorescence microscopy. We found that both anti-parkin and anti-LAMP-3 antibodies strongly co-labelled with pigmented granules (Fig. 4i). Of note, LAMP homologues have been shown to promote 
long term storage of melanin in human keratinocytes ${ }^{22}$; moreover, variants at the LAMP-3 locus have recently been linked to PD susceptibility ${ }^{23}$.

Using confocal microscopy, we demonstrated that in adult midbrain sections anti-parkin signals and neuromelanin granules were frequently surrounded by anti-LAMP-3 reactivity (Fig. $\mathbf{4 j}$ ). We concluded that parkin metabolism is likely associated with LAMP-3-mediatedsequestration of DA radicals in the $S$. nigra of human midbrain.

\section{DISCUSSION}

We identified a heretofore unrecognized role for parkin in redox homeostasis of human brain. To date, only one other protein reportedly has a similar duality in functions (i.e., ubiquitylation and redox biology), namely the sensitive-to-apoptosis gene product, SAG (aka RBX2/ROC2/RNF7) ${ }^{24}$. Our work establishes that parkin's progressive insolubility in human brain, first described in the cortex by Pawlyk et al. ${ }^{17}$, is due to oxidative modifications during normal ageing (Fig. 1, 2d-k; Extended Data Table 1). Unlike in adult brain, where parkin is mostly insoluble after the fourth decade, in the human spinal cord and aged skeletal muscle $\sim 50 \%$ of detectable parkin remains soluble. In all other aged mammals we examined, brain parkin remains soluble to an even greater degree (Fig. 1i; Extended Data Fig. 1.e,f). Theoretically, other modifications (e.g., phosphorylation ${ }^{25}$ ) could also alter parkin's solubility.

Heretofore, parkin oxidation in cells has been interpreted as a 'loss-of-its-function' in vivo. In contrast, we posit that parkin's post-translational oxidation, related aggregate formation, and translocation into lysosomes all reflect its physiological function to maintain redox balance. Ubiquitylation and redox stabilization may not be mutually exclusive. Low concentrations of pro-oxidants have been shown to initiate parkin's ubiquitin ligase activity in vitro ${ }^{15}$, which we confirmed $^{\&}$.

The cellular thiol network is critical in DA cell health ${ }^{26}$. As summarized in Extended Data Fig. 9, through its oxidation we postulate that parkin neutralizes $\mathrm{ROS}$ (e.g., $\mathrm{H}_{2} \mathrm{O}_{2}$ to water; Fig. 3), reduces RNS, conjugates RES (e.g., quinones), promotes melanin formation, and sequesters radicals within insoluble aggregates (Fig. 4). This multimodal redox function is supported three-fold. One, in mice by evidence of sustained oxidative stress in parkin's absence $^{6,7}$. Two, in humans by complementary findings in ARPD brain (Fig. 3) and the observation of lesser neuromelanin content in surviving DA neurons ${ }^{27-30,51-52}$. We noted with interest that primate-specific residue C95 (located outside of its RING domains) was most frequently found to conjugate DA radicals (Fig. 4g; Extended Data Fig. 6c). And three, in parallel work, we discovered a dynamic feedback loop between PRKN expression and glutathione levels in vivo ${ }^{\&}$.

We found that single point mutants have measurable redox effects depending on the experimental context explored (Fig. 3, 4). Such complementary studies will inform clinical genotype-to-phenotype studies in humans ${ }^{31,32}$. Several reports have shown that point mutation carriers have a later age-of-onset of ARPD than those whose alleles encode no RING domain $^{31,32}$. The subcellular translocation of parkin (to mitochondria or lysosomes) could be

\footnotetext{
\&El-Kodsi D et al., Parkinson Disease-Linked Parkin Lowers Cytosolic Oxidative Stress in Feedback Loop with Glutathione (2020), manuscript in preparation
} 
facilitated by oxidation-induced multimer formation at its RING domains (Fig. 2g,h). This concept of 'RING scaffolding' has previously been described for other ubiquitin ligases ${ }^{33}$. Because neuronal health and axonal integrity are co-regulated by redox chemistry ${ }^{34}$, we posit that parkin confers its 'inherently present, pro-mitochondrial benefit' ${ }^{35}$ via redox homeostasis.

Our study raises new questions. Beyond its localization within LAMP- $3^{+}$-lysosomes in DA cells (Fig. 4i,j), our results have not yet revealed the fate of oxidized parkin in other neurons. Human LAMP proteins have been shown to store melanin in human keratinocytes to protect them from UV light ${ }^{22}$. Thus, oxidized parkin together with LAMP-3 (and possibly, LAMP-2) could play a similar role in the ongoing storage of neuromelanin and other radicals, thus facilitating their effective sequestration in ageing brain. Of note, the three clones that detect parkin by microscopy seem to preferentially bind to oxidized/aggregated forms in situ. Little anti-parkin signal was detected in midbrain sections from young adults (Extended Data Fig. 8d), whereas the holoprotein was readily detected by immunoblotting (e.g., Fig. 1a).

Several limitations were identified. One, although we have detected the $P R K N$ gene to be continuously transcribed in nigral neurons, even at age 99 years (Fig. 1e), the rate of its mRNA translation under redox stress conditions, such as during ageing, remains to be studied. Future studies will employ sensitive ELISA-based techniques to quantify the precise concentration of nascent parkin in tissues. Two, we have only begun to map parkin's oxidative modifications that occur in normal human brain (e.g., Extended Data Table 1). As highlighted in Extended Data Fig. 3, parkin likely carries a heterogenous complement of modified cysteine residues in human brain, making the completion of qualitative mapping by LC-MS/MS difficult. Moreover, ROS and RNS modifications can be very transient and thus difficult to capture directly ${ }^{15,16,36}$. Three, we have not yet completed a quantitative assessment of oxidative modifications in young vs. old human brain extracts. Future MS studies require further optimization and larger amounts of human tissues including from healthy midbrains. In addition, access to midbrain organoids ${ }^{37}$, such as those generated from entirely parkin-deficient patients and subjects carrying $P R K N$ point mutants, will provide clarification as to which parkin domains are associated with neuromelanin formation.

The strength of this study is that it focused on parkin metabolism in human brain. $P R K N$ linked ARPD is a human condition, and we are guided by insights from human studies. We posit that parkin fulfils three criteria of an essential redox molecule: the sensing of stressors; structural responses to them; and the participation in corrective measures. If confirmed by future work, this new concept for parkin's function in the human brain opens the door to revisit a possible role in non- $P R K N$-linked PD and in parkinsonism associated with advanced ageing. It also allows the revisiting of therapeutic approaches in patients afflicted by ARPD, such as through viral vector delivery of $P R K N$ cDNA into parkin-deficient brain ${ }^{38}$ and/or by polyvalent, anti-oxidant therapy in select patients ${ }^{39}$. 


\section{MATERIALS AND METHODS}

\section{Tissue collection}

All tissue was collected in accordance with Institutional Review Board-approved guidelines. Fresh frozen samples of cortical human brain from subjects under 50 years of age were acquired through the University of Alabama and the Autism Tissue Program. Post mortem, frozen brain samples from frontal cortices were also obtained from the NICHD Brain and Tissue Bank at the University of Maryland. Brain tissues, including midbrain specimens, with short PMI were also obtained from patients diagnosed with clinical and neuropathological MS according to the revised 2010 McDonald's criteria $(n=4){ }^{40}$. Tissue samples were collected from MS patients with full ethical approval (BH07.001) and informed consent as approved by the CRCHUM research ethics committee. Autopsy samples were preserved and lesions classified using Luxol Fast Blue / Haematoxylin \& Eosin staining and Oil Red O staining as previously published ${ }^{41,42}$. No inflamed tissue areas were used in this current study. Additional, fresh-frozen and paraffinembedded human samples were obtained from the Neuropathology Service at Brigham and Women's Hospital and from archived autopsy specimens in the Department of Pathology and Laboratory Medicine of The Ottawa Hospital. Human spinal cord and muscle tissues were collected at The Ottawa Hospital with approval from the Ottawa Health Science Network Research Ethics Board.

Wild-type $\mathrm{C} 57 \mathrm{Bl} / 6 \mathrm{~J}$ mice were used for analysis of the effects of post mortem interval on murine parkin in the brain. Mice from 4 to 8 months old were perfused with PBS and their brains were collected for PMI experiments.

\section{Sequential extraction of parkin from tissue}

Roughly $1 \mathrm{~cm}^{3}$ samples of human brain frontal cortex and midbrain (age range 5-85 years of age) were weighed and placed in $3 X$ volume/weight of Tris Salt buffer (TSS) (5mM Tris, 140 $\mathrm{mM} \mathrm{NaCl} \mathrm{pH} 7.5$ ) containing complete EDTA-free protease inhibitor cocktail, and $10 \mathrm{mM}$ iodoacetamide (IAA). The samples were homogenized on ice in a Dounce glass homogenizer by 50 passes, transferred to ultracentrifuge tubes and spun at 55,000 rpm and $4^{\circ} \mathrm{C}$ for $30 \mathrm{mins}$. The TS supernatant was transferred to a fresh tube and the pellet was extracted further with addition of $3 x$ volume/weight of Triton X-100 buffer (TX, TS $+2 \%$ Triton X-100). The samples were mixed by vortex, incubated on ice for $10 \mathrm{~min}$ and centrifuged again using the same prior setting. The TX supernatant was transferred to a fresh tube and the pellet was extracted further with addition of $3 x$ volume/weight of SDS buffer (SDS, TS $+2 \%$ SDS). The samples were mixed by vortex, incubated at room temperature for $10 \mathrm{~min}$ and centrifuged again at $55,000 \mathrm{rpm}$ and $12^{\circ} \mathrm{C}$ for 30 mins. The SDS supernatant was transferred to a fresh tube and the pellet was either stored at $-80^{\circ} \mathrm{C}$ or extracted further with addition of $3 \mathrm{X}$ volume/weight of $6 \mathrm{X}$ non-reducing Laemmli buffer (LB, $30 \%$ SDS, $60 \%$ glycerol, $0.3 \%$ bromophenol blue, $0.375 \mathrm{M}$ Tris. pH $6.8,100 \mathrm{mM}$ DTT), mixed by vortex and incubated at room temperature for $10 \mathrm{~min}$. Samples were centrifuged again at 55,000 rpm and $12^{\circ} \mathrm{C}$ for 30 mins and the LB supernatant was transferred to a fresh tube. Extracted proteins from TS, TXS and SDS buffers including pellet (20-30 $\mu \mathrm{g})$ and 10-20 $\mu \mathrm{L}$ of LB extracts were run on SDS-PAGE using reducing (100 mM dithiothreitol, DTT) and/or non-reducing (0 mM DTT) loading buffer. Following transfer to membranes, Ponceaus $\mathrm{S}$ 
staining was used to confirm loading, and samples were blotted for parkin (Biolegend 808503, 1: 5,000), DJ-1 (ab18257, 1: 2,000), $\alpha$-synuclein (syn1 1:1,000 or MJFR1 1:2000), LC3B (3868 1:2000), VDAC (MSA03 1:5000), MnSOD and GLO1 (1:1000), calnexin (MAB3126), cathepsin D (sc-6486), GRP75 (sc-1058) . ImageJ software (1.52 k, National Institutes of Health, USA) was used for signal quantification purposes.

\section{mRNA Analysis}

PRKN mRNA isolated from individual S. nigra DA neurons (SNDA), cortical pyramidal neurons (PY) and non-neuronal, blood mononuclear cells (NN) were processed, as described ${ }^{43}$ and as annotated in the Human BRAINcode database (www.humanbraincode.org).

\section{$\operatorname{ROS}\left(\mathrm{H}_{2} \mathrm{O}_{2}\right)$ measurements}

Amplex ${ }^{\circledR}$ red hydrogen peroxide/peroxidase assay kit (Invitrogen A22188) was used to monitor endogenous levels of $\mathrm{H}_{2} \mathrm{O}_{2}$. Pre-weighed cortex pieces from human brains were homogenized on ice in the $1 \mathrm{x}$ reaction buffer provided, using a Dounce homogenizer ( 3 times volume to weight ratio). Homogenates were diluted in the same $1 \mathrm{x}$ reaction buffer $(10 \mathrm{x}$ and $5 \mathrm{x})$. A serial dilution of the $\mathrm{H}_{2} \mathrm{O}_{2}$ standard provided was prepared $(20,10,2$ and $0 \mu \mathrm{M}) .50 \mu \mathrm{L}$ of standards and samples were plated in a 96 well black plate with clear flat bottom. The reaction was started by the addition of $50 \mu \mathrm{L}$ working solution which consist of $1 \mathrm{x}$ reaction buffer, Amplex ${ }^{\circledR}$ red and horseradish peroxidase. The plate was incubated at room temperature for 30 minutes protected from light. A microplate reader was used to measure either fluorescence with excitation at 560 $\mathrm{nm}$ and emission at $590 \mathrm{~nm}$, or absorbance at $560 \mathrm{~nm}$. The obtained $\mathrm{H}_{2} \mathrm{O}_{2}$ levels $(\mu \mathrm{M})$ were normalized to the tissue weight $(\mathrm{g})$.

\section{Recombinant protein expression in PET-SUMO vector}

Wild-type and truncated (residues 321-465) human parkin were expressed as 6His-Smt3 fusion proteins in Escherichia coli BL21 (DE3) Codon-Plus RIL competent cells (C2527, New England Biolabs) as previous described ${ }^{44-46}$. DJ-1 and SNCA coding sequences were cloned from a pcDNA3.1 vector into the pET-SUMO vector using PCR and restriction enzymes. ARPDassociated parkin mutants in the pET-SUMO vector were generated using site-directed mutagenesis. All proteins were overexpressed in Escherichia coli BL21 Codon-Plus competent cells (C2527, New England Biolabs) and grown at $37^{\circ} \mathrm{C}$ in $2 \%$ Luria Broth containing $30 \mathrm{mg} / \mathrm{L}$ kanamycin until OD600 reached 0.6, at which point the temperature was reduced to $16^{\circ} \mathrm{C}$. All Parkin-expressing cultures were also supplemented with $0.5 \mathrm{mM} \mathrm{ZnCl}_{2}$. Once OD600 reached 0.8 , protein expression was induced with isopropyl $\beta$-D-1-thiogalactopyranoside, except ulp 1 protease, which was induced once OD600 had reached 1.2. The concentration of IPTG used for each construct is as follows: $25 \mu \mathrm{M}$ for wild-type and point mutants of parkin, and $0.75 \mathrm{mM}$ for truncated parkin, DJ-1, $\alpha$-synuclein, SAG, and ulp1 protease. Cultures were left to express protein for 16-20 h. Cells were then harvested, centrifuged, lysed and collected on Ni-NTA agarose beads in elution columns. 


\section{Protein redox chemistry and oxidative toxin conjugation}

The recombinant protein samples were first prepared by removing excess TCEP, present in the elution buffer using repeat centrifugations ( 8 times $4000 \mathrm{x}$ g at $4^{\circ} \mathrm{C}$ for $10 \mathrm{~min}$ ) in Amicon Ultra $10 \mathrm{kDa}$ MWCO filters. The protein concentrations were measured and adjusted to $20 \mu \mathrm{M}$. Stock solutions of rotenone $(1 \mathrm{mM})$, carbonyl cyanide m-chlorophenyl hydrazone (CCCP, $50 \mathrm{mM})$, and hydrogen peroxide $\left(\mathrm{H}_{2} \mathrm{O}_{2}, 9.8 \mathrm{mM}\right)$ were prepared. Aminochrome was freshly synthesized from Dopamine (see below). An aliquot of $10 \mu \mathrm{L}$ of each protein sample (at $20 \mu \mathrm{M}$ ) was reacted with oxidants at the following concentrations: $0,10,20,200,2000 \mu \mathrm{M}$ rotenone; $0,10,20,200$ $\mu \mathrm{M}$ CCCP; 0, 2, 20, 50, 200 aminochrome; 0, 20, 200, 500, 750, 1000, $2000 \mu \mathrm{M} \mathrm{H}_{2} \mathrm{O}_{2}$ or 0, 10, $50,100,200,500,1,000 \mu \mathrm{M}$ DTT. The samples were treated for $30 \mathrm{~min}$ at $37^{\circ} \mathrm{C}$ and centrifuged at $14,000 \mathrm{rpm}$ for $15 \mathrm{~min}$. The supernatant was transferred to a fresh tube and the remaining pellet was extracted with $10 \mu \mathrm{L}$ of T200-TCEP containing either $10 \%$ SDS or $100 \mathrm{mM}$ DTT. The pellets were incubated again for $30 \mathrm{~min}$ at $37^{\circ} \mathrm{C}$ and centrifuged at $14,000 \mathrm{rpm}$ for $15 \mathrm{~min}$. Laemmli buffer ( $10 \mu \mathrm{L}$, containing $100 \mathrm{mM}$ mercaptoethanol) was added to both the pellet and supernatant fractions and samples were separated on two SDS-PAGE. One gel was used for ingel protein staining and the other was used for NBT staining. Specific bands of aminochrome treated wild-type full length r-parkin were excised from silver-stained gels and analyzed by LCMS/MS as described below.

\section{Aminochrome synthesis}

A solution of $0.1 \mathrm{M}$ sodium phosphate buffer $\mathrm{pH} 6.0$ was prepared from a mixture of $12 \mathrm{~mL}$ of $1 \mathrm{M} \mathrm{NaH}_{2} \mathrm{PO}_{4}$ and $88.0 \mathrm{~mL}$ of $1 \mathrm{M} \mathrm{Na}_{2} \mathrm{HPO}_{4}$. The reaction buffer $(0.067 \mathrm{M}$ sodium phosphate, pH 6.0) was prepared by adding $33 \mathrm{~mL}$ of $0.1 \mathrm{M}$ sodium phosphate buffer to $17 \mathrm{~mL}$ water. A solution of $10 \mathrm{mM}$ dopamine in reaction buffer was prepared by adding $19 \mathrm{mg}$ of dopamine hydrochloride to $1 \mathrm{~mL}$ of reaction buffer. Oxidation was activated by adding $5 \mu \mathrm{L}$ of tyrosinase $(25,000 \mathrm{U} / \mathrm{mL})$ and the mixture was incubated at room temperature for $5 \mathrm{~min}$. The tyrosinase was separated from the oxidized dopamine using a $50 \mathrm{kDa}$ cut-off Amicon Ultra centrifugation filter by centrifuging at $14,000 \mathrm{rpm}$ for $10 \mathrm{~min}$. The absorbance of the filtrate was measured at a wavelength of $475 \mathrm{~nm}$ using Ultrospec 21000 pro spectrophotometer and the concentration of aminochrome was determined using the Beer-Lambert equation and extinction coefficient of $3058 \mathrm{~L} \mathrm{x} \mathrm{mol}^{-1} \mathrm{x} \mathrm{cm}^{-1}$.

\section{Protein staining methods}

All proteins were separated on pre-cast 4-12\% Bis-Tris SDS-PAGE gels (NPO321BOX, NPO322BOX, NPO336BOX) from Invitrogen using MES running buffer (50mM MES, 50mM Tris, $1 \mathrm{mM}$ EDTA and $0.1 \%$ SDS, pH 7.3) and Laemmli loading buffer (10\% SDS, 20\% glycerol, $0.1 \%$ bromophenol blue, $0.125 \mathrm{M}$ Tris HCl, 200mM DTT or $\beta$-mercaptoethanol). Proteins were stained in gel using SilverQuest ${ }^{\mathrm{TM}}$ Silver Staining Kit (LC6070) from Invitrogen or Coomassie brilliant blue R-250 dye (20278) from ThermoFisher Scientific using the following protocol: The gel was transferred to a plastic container and rocked for $30 \mathrm{~min}$ in Fix Solution (10\% acetic acid, $50 \%$ methanol), followed by staining for $2-24 \mathrm{~h}(0.25 \%$ Coomassie R250) until 
the gel turned a uniform blue. The stain was replaced with Destain Solution $7.5 \%$ acetic acid and 5\% methanol) and the gel was rocked until crisp blue bands appeared. Following a wash with water the gel was stored in $7 \%$ acetic acid. Proteins transferred to PVDF (1620177, BioRad) membranes were stained with Ponceau S solution for $20 \mathrm{~min}$, washed three times with water, imaged and then destained with $0.1 \mathrm{M} \mathrm{NaOH}$ prior to Western blotting. Proteins conjugated to redox-cycling molecules were stained using the following NBT protocol: Following Ponceau S staining and $\mathrm{NaOH}$ destaining, the membrane was washed twice with water, covered from light and incubated with rocking for $45 \mathrm{~min}$ in NBT solution [8-9 $\mathrm{mg}$ of NBT added to $14 \mathrm{~mL}$ of $2 \mathrm{M}$ potassium glycinate buffer $(75 \mathrm{~g}$ glycine in $0.4 \mathrm{~L}$ water and $\mathrm{pH}$ adjusted to 10.0 with $\mathrm{KOH})]$. The membrane was washed twice with destain solution $(0.16 \mathrm{M}$ sodium borate) and incubated in fresh destain solution for 16-24 h. The membrane was washed once with water and imaged before drying.

\section{Dynamic light scattering (DLS)}

The protein buffer (50mM Tris, $200 \mathrm{mM} \mathrm{NaCl}$ and $250 \mu \mathrm{M}$ TCEP, $\mathrm{pH} 7.5$ ) was exchanged for a $20 \mathrm{mM}$ phosphate buffer with $10 \mathrm{mM} \mathrm{NaCl}$ (pH 7.4). $20 \mu \mathrm{M}$ full-length wild-type r-parkin was centrifuged at $14,000 \mathrm{rpm}$ for $60 \mathrm{~min}$ at $4{ }^{\circ} \mathrm{C}$ and light scattering intensity of the supernatant was collected 30 times at an angle of $90^{\circ}$ using a $10 \mathrm{sec}$ acquisition time. Measurements were taken at $37^{\circ} \mathrm{C}$ using a Malvern Zetasizer Nano ZS instrument equipped with a thermostat cell. The correlation data was exported and analyzed using the nanoDTS software (Malvern Instruments). The samples were measured at 0-, 1-, 3- and 5 hours. Following $24 \mathrm{hr}$ incubation, $2 \mathrm{mM}$ DTT was added to the sample and the light scattering intensity of the supernatant was measured again.

\section{Far UV circular dichroism spectroscopy}

$15 \mu \mathrm{M}$ of reduced and partially oxidized full-length wild-type r-parkin was measured at $\mathrm{t}=0$ and $\mathrm{t}=5$ days of incubation under native conditions in $20 \mathrm{mM}$ phosphate, $10 \mathrm{mM} \mathrm{NaCl}$ buffer. The aggregates rich phase and the monomer rich phase in the samples were separated with ultracentrifugation (100,000 g for 2 hours). Far UV circular dichroism (CD) spectra were recorded for the monomer and aggregated rich phase of protein samples using a JASCO J-720 spectrometer. The final spectrum was taken as a background-corrected average of 5 scans carried out under the following conditions: wavelength range $250-190 \mathrm{~nm}$ at $25{ }^{\circ} \mathrm{C}$; bandwidth was 1 $\mathrm{nm}$; acquisition time was $1 \mathrm{sec}$ and intervals was $0.2 \mathrm{~nm}$. Measurements were performed in a $0.01 \mathrm{~cm}$ cell. CD spectra were plotted in mean residue molar ellipticity units $\left(\mathrm{deg} \mathrm{cm}^{2} \mathrm{dmol}^{-1}\right)$ calculated by the following equation: $[\Theta]=\Theta_{\text {obs }} /(10 \mathrm{ncl})$, where $[\Theta]$ is the mean residue molar ellipticity as a function of wavelength, $\Theta_{\text {obs }}$ is the measured ellipticity as a function of wavelength (nm), $n$ is the number of residues in the protein, $c$ is the concentration of the protein $(\mathrm{M})$, and $l$ is the optical path length $(\mathrm{cm})$. Secondary structure analysis of proteins using CD spectroscopic data was carried out using the BeStSel (Beta Structure Selection) software ${ }^{47,48}$. 


\section{Chemiluminescence/ direct reactive oxygen species (ROS) assay}

The assay was modified from Muller et al. 2013 to measure the ROS-quenching ability of Parkin proteins, DJ-1, SNCA, BSA, GSH, and catalase. Protein concentrations were quantified using Bradford assay and adjusted to 5,10,15 and $30 \mu \mathrm{M}$ in buffer not containing TCEP. BSA (10 and $20 \mu \mathrm{M}), \mathrm{GSH}(15,20,200,400,800$ and $2000 \mu \mathrm{M})$, and catalase $(0.015,0.15,0.25$ and $15 \mu \mathrm{M})$ were prepared. Stock solutions of $\mathrm{H}_{2} \mathrm{O}_{2}$ for standard curve were prepared at 5, 10, 20, 40 and 50 $\mathrm{mM}$ in $0.1 \mathrm{M}$ Tris $\mathrm{HCl} \mathrm{pH} 8.0$ using $30 \% \mathrm{H}_{2} \mathrm{O}_{2}$. Stock solutions of $300 \mathrm{mM}$ luminol and $40 \mathrm{mM}$ 4-iodophenol were prepared in DMSO and protected from light. Signal reagent, containing 1.94 $\mathrm{mM}$ luminol and $0.026 \mathrm{mM}$ 4-iodophenol, was prepared in $0.1 \mathrm{M}$ Tris $\mathrm{HCl} \mathrm{pH} 8.0$ and protected from light. A $0.4 \%$ horseradish peroxidase solution was prepared using HRP-linked anti-rabbit secondary antibody diluted in Stabilizyme solution (SurModics SZ02). Each read was set up in triplicate on a white polystyrene 96-well plate (ThermoFisher 236105) and to each well was added $80 \mu \mathrm{L}$ Stabilizyme, $15 \mu \mathrm{L}$ of $0.4 \%$ horseradish peroxidase (HRP) and $25 \mu \mathrm{L}$ of sample or controls. One of the injectors in a Synergy H1Multi-Mode Plate Reader (Bio Tek) was primed and set to inject $15 \mu \mathrm{L}$ of signal reagent and $15 \mu \mathrm{L}$ of each $\mathrm{H}_{2} \mathrm{O}_{2}$ stock solution was manually added to corresponding controls and samples just prior to reading. Final concentrations of reagents were $0.04 \% \mathrm{HRP}, 500,1000,2000,4000$ and $5000 \mu \mathrm{M} \mathrm{H}_{2} \mathrm{O}_{2}, 194 \mu \mathrm{M}$ luminol, $2.6 \mu \mathrm{M}$ 4 -iodophenol and $0.8,1.7,2.5$ or $5 \mu \mathrm{M}$ of protein. The plate reader was set to measure luminescence every $1 \mathrm{~min}$ for a total of $10 \mathrm{~min}$. The resulting kinetic data was converted to area under the curve (AUC) using Prism version 6. For samples pre-incubated with $20 \mathrm{mM}$ iodoacetamide, a stock solution of $1 \mathrm{M}$ iodoacetamide was prepared. To each well containing 25 $\mu \mathrm{L}$ of sample, $0.52 \mu \mathrm{L}$ of $1 \mathrm{M}$ iodoacetamide and $0.48 \mu \mathrm{L}$ of buffer not containing TCEP was added and the samples were incubated for $2 \mathrm{~h}$ at $37^{\circ} \mathrm{C}$. Following incubation, the reagents for chemiluminescence were added as above except $79 \mu \mathrm{L}$ of Stabilizyme was used instead of $80 \mu \mathrm{L}$ and the samples were analyzed as above.

\section{Thiol quantification of recombinant proteins}

Recombinant protein samples were first prepared by exchanging the T200 protein buffer $(50 \mathrm{mM}$ Tris, $200 \mathrm{mM} \mathrm{NaCl}$ and $250 \mu \mathrm{M}$ TCEP, $\mathrm{pH}$ 7.5) for T200-TCEP using repeat centrifugations (8 times $4000 \mathrm{x}$ g at $4^{\circ} \mathrm{C}$ for $10 \mathrm{~min}$ ) in Amicon Ultra $10 \mathrm{kDa}$ MWCO filters. The protein concentrations were measured and recorded. The glutathione stock solution of $32,539 \mu \mathrm{M}$ was prepared by dissolving $1 \mathrm{mg}$ glutathione (GSH) in $1 \mathrm{~mL}$ of T200-TCEP and the standards 0,50 , $101,203,406,813$ and $1000 \mu \mathrm{M}$ were prepared by serial dilution in T200-TCEP. The reaction buffer (0.1 M sodium phosphate, $\mathrm{pH} 8.0$ ) was prepared by adding $93.2 \mathrm{~mL} 1 \mathrm{M} \mathrm{Na}_{2} \mathrm{HPO}_{4}$ and 6.8 $\mathrm{mL}$ of $\mathrm{NaH}_{2} \mathrm{PO}_{4}$ in $1 \mathrm{~L}$ of water. Thiol detecting reagent (Ellman's reagent) was prepared by dissolving $2 \mathrm{mg}$ of 5,5'-dithio-bis-[2-nitrobenzoic acid] (DNTB) in $1 \mathrm{~mL}$ of reaction buffer. The assay was performed in 96-well clear round bottom plates by adding $50 \mu \mathrm{L}$ of thiol detecting reagent to $50 \mu \mathrm{L}$ of sample or standard and incubating for $15 \mathrm{~min}$ at room temperature. The resulting 5-thio-2-nitrobenzoic-acid (TNB) produced was measured by absorbance at $412 \mathrm{~nm}$ using a Synergy H1Multi-Mode Plate Reader (Bio Tek). The amount of free thiols detected in each sample was calculated using the regression curve obtained from the glutathione standards and dividing by the concentration of the sample. 


\section{Cysteine labeling for mass spectrometry}

The recombinant protein samples were first prepared by exchanging the T200 buffer for PBS. The protein concentrations were measured and adjusted to $10 \mu \mathrm{M}$ using PBS. Stock solutions of $500 \mathrm{mM}$ DTT, $100 \mathrm{mM}$ iodoacetamide (IAA), $100 \mathrm{mM}$ hydrogen peroxide and $250 \mathrm{mM}$ ethylenediaminetetraacetic acid (EDTA) were prepared in PBS. A stock of $500 \mathrm{mM}$ N-ethylmaleimide (NEM) was prepared in ethanol immediately before use. For the first optimization and comparison of IAA and NEM labelling (i.e., Table S2), r-parkin was treated with $2 \mathrm{mM}$ DTT for $30 \mathrm{~min}$ at $37^{\circ} \mathrm{C}$ followed by incubation with $5 \mathrm{mM}$ IAA or $85 \mathrm{mM} \mathrm{NEM}$ for $2 \mathrm{~h}$ at $37^{\circ} \mathrm{C}$. The stepwise cysteine labeling procedure was as follows: A $10 \mu \mathrm{L}$ aliquot of protein (at 10 $\mu \mathrm{M}$ ) was reacted with hydrogen peroxide at various concentrations, as indicated (Table 1) for 30 min (and up to $60 \mathrm{~min}$ ) at $37^{\circ} \mathrm{C}$ as indicated. Any unreacted cysteines were alkylated with incubation with $5 \mathrm{mM}$ IAA (either with or, in some runs, without $10 \mathrm{mM}$ EDTA) for $2 \mathrm{hrs}$ at $37^{\circ} \mathrm{C}$. Previously oxidized cysteines were then reduced by treatment with $40 \mathrm{mM}$ DTT for 30 min at $37^{\circ} \mathrm{C}$. Newly reduced cysteines were alkylated by incubation with $85 \mathrm{mM}$ N-ethyl maleimide (NEM) for $2 \mathrm{hrs}$ at $37^{\circ} \mathrm{C}$. The samples were separated on SDS-PAGE using Laemmli buffer containing $100 \mathrm{mM}$ DTT and proteins visualized using Coomassie staining. Appropriate bands were excised and analyzed by liquid chromatography mass spectrometry (LC-MS/MS).

\section{Protein identification by LC-MS/MS}

Proteomics analysis was performed at the Ottawa Hospital Research Institute Proteomics Core Facility (Ottawa, Canada). Proteins were digested in-gel using trypsin (Promega) according to the method of Shevchenko ${ }^{49}$. Peptide extracts were concentrated by Vacufuge (Eppendorf). LCMS/MS was performed using a Dionex Ultimate 3000 RLSC nano HPLC (Thermo Scientific) and Orbitrap Fusion Lumos mass spectrometer (Thermo Scientific). MASCOT software version 2.6.2 (Matrix Science, UK) was used to infer peptide and protein identities from the mass spectra. For detection of dopamine metabolites on Parkin, the following variable modifications were included: 5,6-indolequinone $\left(+\mathrm{C}_{8} \mathrm{O}_{2} \mathrm{NH}_{3}, \mathrm{~m} / \mathrm{z}\right.$ shift +145$)$, aminochrome $\left(+\mathrm{C}_{8} \mathrm{O}_{2} \mathrm{NH}_{5}\right.$, $+147)$, aminochrome $+2 \mathrm{H}\left(+\mathrm{C}_{8} \mathrm{O}_{2} \mathrm{NH}_{7},+149\right)$, and dopamine quinone $\left(+\mathrm{C}_{8} \mathrm{O}_{2} \mathrm{NH}_{9},+151\right)$. These samples were prepared for analysis without any use of dithiothreitol or iodoacetamide. The observed spectra were matched against human sequences from SwissProt (version 2018-05) and also against an in-house database of common contaminants. The results were exported to Scaffold (Proteome Software, USA) for further validation and viewing. Analysis of the holoprotein and of three runs of $\mathrm{H}_{2} \mathrm{O}_{2}$-exposed r-parkin (Supplemental Table 2) were performed at the University of Western Ontario. There, samples were run on a QToF Ultima mass spectrometer (Waters) equipped with a Z-spray source and run in positive ion mode with an Agilent 1100 HPLC used for LC gradient delivery (University of Western Proteomics Facility).

\section{MaxQuant analysis of mass spectrometry data}

For applicable experiments, the raw MS data files were further processed with MaxQuant software version 1.6.5 and searched with the Andromeda search engine ${ }^{18}$. The reference fastas were set to uniprot-human (version 2019-02-12) and uniport-ecoli. The E. coli proteome was 
included to account for bacterial proteins present in the recombinant protein samples. The 'second peptides' and 'match between runs' settings were enabled. All other settings were left as default. Selected variable modifications included oxidation (Met), acetylation (protein Nterminus), and carbamidomethyl (Cys), as well as custom modifications for pyrocarbamidomethyl (N-terminal Cys), N-ethylmaleimide (Cys), and NEM+water (Cys). For data analysis, site-level intensity values were obtained from the MaxQuant-generated "CarbamidomethylSites" table which combines the intensity of MS1 signals from all peptides covering a particular cysteine residue.

\section{Immunoprecipitation (IP) of brain parkin}

Conjugation of anti-parkin antibody (Prk8, 808503, lot B209868) to magnetic beads at a final concentration of $10 \mathrm{mg}$ of antibody/ $\mathrm{mL}$ of beads was done following the Magnetic Dynabeads Antibody Coupling Kit from Invitrogen (14311D). Human tissue lysates were also prepared using the "Sequential Extraction of Proteins from Tissue" protocol as described above with addition of $10 \mathrm{mM}$ iodoacetamide prior to homogenization. TS tissue extracts $(n=4)$ and SDS tissue extracts $(n=8)$ were diluted in TS buffer, resulting in a final SDS concentration of 0.0175 $\%$ and $0.05 \%$ respectively. For the IP, Prk 8 conjugated agarose beads were first prepared by multiple washes with $1 \mathrm{~mL}$ of TS buffer using centrifugation $\left(1000 \mathrm{xg}\right.$ at $4{ }^{\circ} \mathrm{C}$ for $\left.3 \mathrm{~min}\right)$ and adhesion to a strong magnet. Amounts of Prk8 conjugated agarose beads used for each experiment were approximated based on the amount of parkin $(\mu \mathrm{g}) /$ sample calculated by densitometry when the sample was compared to recombinant parkin protein standards using Western blotting with Prk8 primary antibody. The mixture was incubated for $16 \mathrm{~h}$ at $4^{\circ} \mathrm{C}$ with slow rotation. Unbound proteins, which did not bind to the Prk8 conjugated agarose beads, were separated from the beads by centrifugation $\left(1000 \mathrm{x} g\right.$ at $4^{\circ} \mathrm{C}$ for $\left.3 \mathrm{~min}\right)$ followed by adhesion to a strong magnet and saved as the IP "unbound" fraction. Beads from cellular or human IP were washed three times with 900 or $1000 \mu \mathrm{L}$ respectively of ice-cold RIPA buffer $(1 \%$ nonionic polyoxyethylene-40, $0.1 \% \mathrm{SDS}, 50 \mathrm{mM}$ Tris, $150 \mathrm{mM} \mathrm{NaCl}, 0.5 \%$ sodium deoxycholate, 1 $\mathrm{mM}$ EDTA) using centrifugation $\left(1000 \mathrm{x} \mathrm{g}\right.$ at $4^{\circ} \mathrm{C}$ for $\left.3 \mathrm{~min}\right)$ and adhesion to a strong magnet. Approximately 5- $10 \mu \mathrm{L}$ of each wash was combined and saved as the IP "wash" fraction. To elute Prk8 bound proteins, 15-35 uL of 6X reducing Laemmli buffer (30 \% SDS, $60 \%$ glycerol, $0.3 \%$ bromophenol blue, $0.375 \mathrm{M}$ Tris, $100 \mathrm{mM}$ DTT, pH 6.8) was added to the beads and the samples were boiled for $5 \mathrm{~min}$. Following centrifugation $\left(1000 \mathrm{x}\right.$ g at $4^{\circ} \mathrm{C}$ for $3 \mathrm{~min}$ ), the supernatant was transferred to a fresh tube labeled "IP elute" and the beads were discarded. To assess IP efficiency, eluted fractions (IP elute), along with controls (input, unbound, wash and recombinant parkin protein standards) were run on SDS/PAGE and blotted with anti-parkin (MAB5512 or 2132S). Human IP elutes used in subsequent for mass spectrometry (MS) analysis were incubated with $500 \mathrm{mM} \mathrm{N}$-ethyl maleimide (as indicated for select runs) for $16 \mathrm{~h}$ at $4^{\circ} \mathrm{C}$ prior to SDS-PAGE and further processing for MS (as described above). Gel slices corresponding to band sizes $50-75 \mathrm{kDa}$ were excised and analyzed by LC-MS/MS.

\section{In vitro melanin formation assay}

The recombinant protein samples were first prepared by exchanging the T200 protein buffer (50 $\mathrm{mM}$ Tris, $200 \mathrm{mM} \mathrm{NaCl}$ and $250 \mu \mathrm{M}$ TCEP, pH 7.5) for T200-TCEP (50 mM Tris and $200 \mathrm{mM}$ $\mathrm{NaCl}, \mathrm{pH} 7.5$ ) using repeat centrifugations ( 8 times $4000 \mathrm{xg}$ at $4^{\circ} \mathrm{C}$ for $10 \mathrm{~min}$ ) in Amicon Ultra 
$10 \mathrm{kDa}$ MWCO filters. The protein concentrations were measured and adjusted to $20 \mu \mathrm{M}$ using T200-TCEP. A $0.067 \mathrm{M}$ sodium phosphate buffer, $\mathrm{pH} 6.0$, was prepared by adding $33 \mathrm{~mL}$ of 0.1 $\mathrm{M}$ sodium phosphate buffer to $17 \mathrm{~mL}$ water and adjusting the $\mathrm{pH}$ using $\mathrm{HCl}$. A stock solution of $100 \mathrm{mM}$ dopamine $\mathrm{HCl}$ was prepared in $0.067 \mathrm{M}$ sodium phosphate buffer and stock solutions of $100 \mathrm{mM}$ reduced glutathione (GSH) and hydrogen peroxide were prepared in T200-TCEP.

Samples and controls were prepared in $100 \mu \mathrm{L}$ total volume and contained: $10 \mu \mathrm{L}$ of $20 \mu \mathrm{M}$ protein or T200-TCEP, $10 \mu \mathrm{L}$ of $100 \mathrm{mM}$ dopamine or $0.067 \mathrm{M}$ sodium phosphate buffer, $10 \mu \mathrm{L}$ of $100 \mathrm{mM}$ glutathione or T200-TCEP buffer, and $70 \mu \mathrm{L}$ T200-TCEP. The final concentration of protein was $2 \mu \mathrm{M}$ and the final concentration of reagents was all $10 \mathrm{mM}$. The samples and controls were plated in triplicate, and absorbance read at 405 and $475 \mathrm{~nm}$ every $90 \mathrm{sec}$ for $1 \mathrm{~h}$. The plate was then protected from light and incubated for $24 \mathrm{~h}$. A final reading of absorbance at 405 and $475 \mathrm{~nm}$ was taken after $24 \mathrm{~h}$.

\section{Cell cytotoxicity assay}

Human neuroblastoma cell line (M17 cells) wild-type, Vector (Myc), P5 (low stable expression of Myc-parkin) and P17 (high stable expression of Myc-parkin) were grown in 6 well culture plates, at $0.3 \times 10^{6}$ cell density (80\% confluence) in Opti-MEM media (Gibco 11052-021) containing heat inactivated FBS (Gibco 10082-147), Pen/strep/Neo (5mg/5mg/10mg) (Gibco 15640-055), MEM non-essential amino acids (10mM) (Gibco 11140-050) and sodium pyruvate $(100 \mathrm{mM})$. For rescue experiments, flag-vector, flag-parkin, flag-p.G328E and flag-p.C431F pcDNA3.1+ were overexpressed in M17 wild-type cells. $4 \mu \mathrm{g}$ of cDNA was transfected using a 1:1 ratio of cDNA: Lipofectamine 2000 (52887, Invitrogen) in OPTI-MEM transfection medium. The cDNA and Lipofectamine 2000 was first incubated for $20 \mathrm{~min}$ at room temperature before being applied directly to the cells for $1 \mathrm{~h}$ at $37^{\circ} \mathrm{C}$ with $5 \% \mathrm{CO}_{2}$ followed by direct addition of fresh growth medium. The cells were incubated another 24 hours at $37^{\circ} \mathrm{C}$ with $5 \% \mathrm{CO}_{2}$.

Dopamine hydrochloride (Sigma H8502) $200 \mathrm{mM}$ stock was prepared. The cells were washed with fresh media once and then incubated with media alone or supplemented with dopamine at final concentrations of $20 \mu \mathrm{M}$ and $200 \mu \mathrm{M}$ for 18-20 hours. Post dopamine stress, media was collected from all wells for cytotoxicity assay, the cells were harvested and lysed with TS buffer and centrifuged. The supernatant was collected and saved for western blot analysis and to assess total cell toxicity signal. The pellet was suspended in SDS buffer and centrifuged.

Vybrant TM cytotoxicity assay kit (Molecular Probes V-23111) was used to monitor cell death through the release of the cytosolic enzyme glucose 6-phosphate dehydrogenase (G6yPD) from damaged cells into the surrounding medium. $50 \mu \mathrm{l}$ of media alone (no cells), media from control and stressed cells and cell lysates were added to a 96-well microplate. Fifty $\mu 1$ of reaction mixture, containing reaction buffer, reaction mixture and resazurin, was added to all wells, and the mircroplate was incubated at $37^{\circ} \mathrm{C}$ for 30 mins. A microplate reader was used to measure either fluorescence with excitation at $560 \mathrm{~nm}$ and emission at $590 \mathrm{~nm}$. A rise in fluoresence indicates a rise in G6PD levels i.e. a rise in cell death. 


\section{Immunohistochemistry (IHC)}

Immunohistochemistry was performed on paraffin-embedded sections and treated as previously described (Schlossmacher et al. 2002; Schlossmacher and Shimura 2005, Shutinoski et al 2019). Briefly, prior to antibody incubation, sections were deparaffinized in xylene and successively rehydrated through a series of decreasing ethanol concentration solutions. Endogenous peroxidase activity was quenched with $3 \%$ hydrogen peroxide in methanol, followed by a standard citric acid-based antigen retrieval protocol to unmask epitopes. Sections were blocked in 10-20\% serum in PBS-T to reduce non-specific signal. Sections were incubated overnight at $4{ }^{\circ} \mathrm{C}$ in primary antibodies diluted in $1-5 \%$ serum in PBS-T according to the following concentrations: novel Parkin mAbs from Biolegend clones D (BioLegend, A15165D; 1:250), clone E (BioLegend, A15165E; 1:2000), and clone G (1:250), PRK8 (BioLegend, MAB5512; 1:500), Lamp3 (Santa Cruz, SC5275; 1:100), LC3B (Sigma, L7543-200uL; 1:100), VDAC (MitoScience, MSA03; 1:100). Biotinylated secondary antibodies (biotinylated anti mouse IgG $(\mathrm{H}+\mathrm{L})$ made in goat; Vector Labs, BA-9200, biotinylated anti-rabbit $\operatorname{IgG}(\mathrm{H}+\mathrm{L})$ made in goat; Vector Labs, BA-1000) were diluted to 1:225 and sections were incubated for 2 hours at room temperature. The signal was amplified with VECTASTAIN® Elite ${ }^{\circledR}$ ABC HRP Kit (Vector Labs, PK-6100), and visualized via standard DAB solution, 55mM DAB, or Vina green (Biocare Medical, BRR807AH), or most commonly metal enhanced DAB (Sigma, SIGMAFAST ${ }^{\mathrm{TM}}$ DAB with Metal Enhancer D0426). Samples were counterstained with Harris Modified Hematoxylin nuclei stain and dehydrated through a series of increasing ethanol concentration solutions and xylene. Permount (Fisher Scientific, SP15-100) was used for mounting and slides were visualized with high magnification images via a Quorum Slide Scanner (Ottawa Hospital Research Institute).

\section{Immunofluorescence (IF) and confocal microscopy}

Paraffin-embedded human midbrain sections were stained by routine indirect immunofluorescence with the following details. Antigen retrieval was performed in Tris-EDTA buffer $\mathrm{pH} 9$ for 10 mins. Primary antibodies were incubated overnight at 4C. Details for primary antibodies anti-Parkin Clone E (1:500), anti-LAMP3 (1:250) are described above. A 40 minute incubation with the following secondary antibodies was performed: goat anti-mouse alexa fluor 488 (1:200), goat anti-rabbit alexa fluor 594 (1:500). Slides were mounted with fluorescence mounting medium with DAPI. Stained sections were imaged using a Zeiss LSM 880 AxioObserver Z1 with an Airyscan Confocal Microscope then processed and analyzed using Zeiss Zen and Fiji software.

\section{Statistical analyses}

All statistical analyses were performed using GraphPad Prism version 6 (GraphPad Software, San Diego, CA, USA, www.graphpad.com). Differences between two groups were assessed using an unpaired t-test. Differences among 3 or more groups were assessed using a one-way or two-way ANOVA followed by Tukey's post hoc corrections to identify statistical significance. Subsequent post hoc tests are depicted graphically and show significance between treatments. 
For all statistical analysis a cut-off for significance was set at 0.05 . Data is displayed with $\mathrm{p}$ values represented as ${ }^{*} \mathrm{p}<0.05,{ }^{* *} \mathrm{p}<0.01,{ }^{* * *} \mathrm{p}<0.001$, and $* * * * \mathrm{p}<0.0001$

Logistic/linear regression modelling: Linear regression (for continuous dependent variable, e.g. $\mathrm{H}_{2} \mathrm{O}_{2}$ level, mRNA level) or logistic regression (for binary dependent variable, e.g., parkin present in TS fraction) modelling were performed. Furthermore, to address the effect of age on parkin solubility, receiver operating characteristic (ROC) curve and area under the ROC curve (AUC) were calculated, as reported ${ }^{50}$.

\section{ACKNOWLEDGMENTS}

We are grateful for the commitment of patients and their family members to participate in autopsy studies. We thank Dr. J. Palacino for creating stable M17 cell lines, Drs. A. Brice and E. Fon for sharing prkn-null mice, Dr. B. Madras for providing specimens of cynomolgus brain, Drs. R. Tam, L. Dong and Ms. K. Solti and Ms. H. Boston for technical support, Dr. D. Gibbings for antibodies, Dr. D. Gray for assistance with confocal imaging, Drs. M. Medina and R. R. Ratan for encouragement, Drs. S. Bennett, D. Pratt for discussions, and Drs. L. Zecca, F. Zucca, H. Lochmueller, M. Rousseaux, and members of the Schlossmacher lab for their critical comments. Funding: This work was supported by the: Parkinson Research Consortium of Ottawa (J.M.T., D.N.E.K., J.J.T.); Queen Elizabeth II Graduate Scholarship Fund (J.M.T.); Government of Canada [NSERC (J.K.); CIHR MD/PhD Program (J.M.T., A.C.N.); CIHR Research Grant (G.S.S., A.P.); CIHR Canada Research Chair Program (M.G.S., A.P.)]; Michael J. Fox Foundation for Parkinson's Research (P.T., J.J.T., M.G.S.); The Research Foundation of the MS Society of Canada; Progressive MS Alliance (A.P.); Hungarian Brain Research Program (G.T.); Uttra and Sam Bhargava Family (E.T., M.G.S.); and The Ottawa Hospital (E.T., M.G.S.). Author contributions: Study design: J.M.T., D.N.E.K., P.T., J.J.T., M.G.S.; Writing and Figure preparation: J.M.T., D.N.E.K., N.A.L., T.K.F., M.J., A.P.N., J.L., G.S.S., J.M.W., G.T., P.T., J.J.T., and M.G.S. prepared the initial draft of the manuscript and figures. All authors reviewed and / or edited the manuscript and approved of the submitted versions. Experiments: J.M.T., D.N.E.K., N.A.L., T.K.F., M.J., A.P.N., B.O., L.W., J.K., A.C.N., Q.J., R.S., J.L., M.Z., K.R.B., A.T., X.D., L.P., G.T. performed experiments; and C.R.S., A.B.W., E.T., A.H., A.P., J.A.C., provided data, tissue specimens and critical comments. Analysis: J.M.T., D.N.E.K., J.L., T.K.F., G.S.S., L.P., G.T., J.M.W., P.T., J.J.T., M.G.S. performed data analyses. Study supervision: P.T., J.J.T., M.G.S. Overall responsibility: M.G.S. This work is dedicated to the memory of Mr. Bruce Hayter (1962-2019), a tireless advocate for persons with young-onset parkinsonism.

Competing interests: Drs. B. O’Nuallain, M. Jin, L. Wang, P. Taylor are (or were) employees of BioLegend Inc. (Dedham, MA., USA). The Ottawa Hospital receives payments from BioLegend Inc. related to licensing agreements for immunological reagents related to parkin and $\alpha$-synuclein. Dr. M. Schlossmacher received travel reimbursements from the Michael J. Fox Foundation for Parkinson's Research for participation in industry summits and consulting fees as well as royalties from Genzyme-Sanofi for patents unrelated to this work. Dr. G. Toth is an 
employee and a shareholder of Cantabio Pharmaceuticals. Dr. A. Holmgren (deceased) served as chairman and senior scientist at IMCO Corporation Ltd AB, Stockholm, Sweden. No additional, potentially competing financial interests are declared.

Data and materials availability: Original data associated with this study are available in the main text and extended data figures and tables; additional data will be made available upon request. 


\section{REFERENCES}

1 Kitada, T. et al. Mutations in the parkin gene cause autosomal recessive juvenile parkinsonism. Nature 392, 605-608, doi:10.1038/33416 (1998).

2 Barodia, S. K., Creed, R. B. \& Goldberg, M. S. Parkin and PINK1 functions in oxidative stress and neurodegeneration. Brain Res Bull 133, 51-59, doi:10.1016/j.brainresbull.2016.12.004 (2017).

3 Zucca, F. A. et al. Neuromelanin of the human substantia nigra: an update. Neurotox Res 25, 13-23, doi:10.1007/s12640-013-9435-y (2014).

4 Carballo-Carbajal, I. et al. Brain tyrosinase overexpression implicates age-dependent neuromelanin production in Parkinson's disease pathogenesis. Nat Commun 10, 973, doi:10.1038/s41467-019-08858-y (2019).

5 Whitworth, A. J. et al. Increased glutathione S-transferase activity rescues dopaminergic neuron loss in a Drosophila model of Parkinson's disease. Proceedings of the National Academy of Sciences 102, 8024-8029, doi:10.1073/pnas.0501078102 (2005).

6 Palacino, J. J. et al. Mitochondrial dysfunction and oxidative damage in parkin-deficient mice. J Biol Chem 279, 18614-18622, doi:10.1074/jbc.M401135200 (2004).

7 Periquet, M., Corti, O., Jacquier, S. \& Brice, A. Proteomic analysis of parkin knockout mice: alterations in energy metabolism, protein handling and synaptic function. $J$ Neurochem 95, 1259-1276, doi:10.1111/j.1471-4159.2005.03442.x (2005).

8 Itier, J. M. et al. Parkin gene inactivation alters behaviour and dopamine neurotransmission in the mouse. Hum Mol Genet 12, 2277-2291, doi:10.1093/hmg/ddg239 (2003).

9 Lee, S. J., Kim, D. G., Lee, K. Y., Koo, J. S. \& Lee, B. J. Regulatory mechanisms of thiol-based redox sensors: lessons learned from structural studies on prokaryotic redox sensors. Arch Pharm Res 41, 583-593, doi:10.1007/s12272-018-1036-0 (2018).

10 Segura-Aguilar, J. et al. Protective and toxic roles of dopamine in Parkinson's disease. $J$ Neurochem 129, 898-915, doi:10.1111/jnc.12686 (2014).

11 LaVoie, M. J., Cortese, G. P., Ostaszewski, B. L. \& Schlossmacher, M. G. The effects of oxidative stress on parkin and other E3 ligases. J Neurochem 103, 2354-2368, doi:10.1111/j.1471-4159.2007.04911.x (2007).

12 Meng, F. et al. Oxidation of the cysteine-rich regions of parkin perturbs its E3 ligase activity and contributes to protein aggregation. Mol Neurodegener 6, 34, doi:10.1186/1750-1326-6-34 (2011).

13 Chung, K. K., Dawson, V. L. \& Dawson, T. M. S-nitrosylation in Parkinson's disease and related neurodegenerative disorders. Methods Enzymol 396, 139-150, doi:10.1016/S00766879(05)96014-X (2005).

14 LaVoie, M. J., Ostaszewski, B. L., Weihofen, A., Schlossmacher, M. G. \& Selkoe, D. J. Dopamine covalently modifies and functionally inactivates parkin. Nat Med 11, 12141221, doi:10.1038/nm1314 (2005).

15 Yao, D. et al. Nitrosative stress linked to sporadic Parkinson's disease: S-nitrosylation of parkin regulates its E3 ubiquitin ligase activity. Proc Natl Acad Sci U S A 101, 1081010814, doi:10.1073/pnas.0404161101 (2004).

16 Chung, K. K. et al. S-nitrosylation of parkin regulates ubiquitination and compromises parkin's protective function. Science 304, 1328-1331, doi:10.1126/science. 1093891 (2004). 
17 Pawlyk, A. C. et al. Novel monoclonal antibodies demonstrate biochemical variation of brain parkin with age. $J$ Biol Chem 278, 48120-48128, doi:10.1074/jbc.M306889200 (2003).

18 Cox, J. \& Mann, M. MaxQuant enables high peptide identification rates, individualized p.p.b.-range mass accuracies and proteome-wide protein quantification. Nat Biotechnol 26, 1367-1372, doi:10.1038/nbt.1511 (2008).

19 Shimura, H. et al. Ubiquitination of a new form of alpha-synuclein by parkin from human brain: implications for Parkinson's disease. Science 293, 263-269, doi:10.1126/science.1060627 (2001).

20 Monzani, E. et al. Dopamine, Oxidative Stress and Protein-Quinone Modifications in Parkinson's and Other Neurodegenerative Diseases. Angew Chem Int Ed Engl 58, 65126527, doi:10.1002/anie.201811122 (2019).

21 Zucca, F. A. et al. Neuromelanin organelles are specialized autolysosomes that accumulate undegraded proteins and lipids in aging human brain and are likely involved in Parkinson's disease. NPJ Parkinsons Dis 4, 17, doi:10.1038/s41531-018-0050-8 (2018).

22 Correia, M. S. et al. Melanin Transferred to Keratinocytes Resides in Nondegradative Endocytic Compartments. J Invest Dermatol 138, 637-646, doi:10.1016/j.jid.2017.09.042 (2018).

23 Zimprich, A. Genetics of Parkinson's disease and essential tremor. Curr Opin Neurol 24, 318-323, doi:10.1097/WCO.0b013e3283484b87 (2011).

24 Sun, Y. \& Li, H. Functional characterization of SAG/RBX2/ROC2/RNF7, an antioxidant protein and an E3 ubiquitin ligase. Protein Cell 4, 103-116, doi:10.1007/s13238-0122105-7 (2013).

25 Koyano, F. et al. Ubiquitin is phosphorylated by PINK1 to activate parkin. Nature 510, 162-166, doi:10.1038/nature13392 (2014).

26 Go, Y. M., Chandler, J. D. \& Jones, D. P. The cysteine proteome. Free Radic Biol Med 84, 227-245, doi:10.1016/j.freeradbiomed.2015.03.022 (2015).

27 Gibb, W. R., Narabayashi, H., Yokochi, M., Iizuka, R. \& Lees, A. J. New pathologic observations in juvenile onset parkinsonism with dystonia. Neurology 41, 820-822, doi:10.1212/wnl.41.6.820 (1991).

28 Takahashi, H. et al. Familial juvenile parkinsonism: clinical and pathologic study in a family. Neurology 44, 437-441, doi:10.1212/wnl.44.3_part_1.437 (1994).

29 Yamamura, Y., Kuzuhara, S, Kondo, K., Yanagi, T., Uchida, M, Matsumine, H., \& Mizuno, Y., Clinical, pathological and genetic studies on autosomal recessive early-onset parkinsonism with diurnal fluctuation. Parkinsonism Relat Disord. 4, 65-72 (1998).

30 Hayashi, S. et al. An autopsy case of autosomal-recessive juvenile parkinsonism with a homozygous exon 4 deletion in the parkin gene. Mov Disord 15, 884-888 (2000).

31 Farrer, M. et al. Lewy bodies and parkinsonism in families with parkin mutations. Ann Neurol 50, 293-300 (2001).

32 Pramstaller, P. P. et al. Lewy body Parkinson's disease in a large pedigree with 77 Parkin mutation carriers. Ann Neurol 58, 411-422, doi:10.1002/ana.20587 (2005).

33 Kentsis, A., Gordon, R. E. \& Borden, K. L. Control of biochemical reactions through supramolecular RING domain self-assembly. Proc Natl Acad Sci U S A 99, 15404-15409, doi:10.1073/pnas.202608799 (2002). 
34 Norton, M. et al. ROMO1 is an essential redox-dependent regulator of mitochondrial dynamics. Sci Signal 7, ra10, doi:10.1126/scisignal.2004374 (2014).

35 Berger, A. K. et al. Parkin selectively alters the intrinsic threshold for mitochondrial cytochrome c release. Hum Mol Genet 18, 4317-4328, doi:10.1093/hmg/ddp384 (2009).

36 Vandiver, M. S. et al. Sulfhydration mediates neuroprotective actions of parkin. Nat Commun 4, 1626, doi:10.1038/ncomms2623 (2013).

37 Mohamed, N., Mathur, M., da Silva, R. V., Beitel, L. K., Fon, E. A., Durcan, T. M. Generation of human midbrain organoids from induced pluripotent stem cells. MNI Open Res 3, doi:https://doi.org/10.12688/mniopenres.12816.1 (2019).

38 Kitada, T., Tomlinson, J. J., Ao, H. S., Grimes, D. A. \& Schlossmacher, M. G. Considerations regarding the etiology and future treatment of autosomal recessive versus idiopathic Parkinson disease. Curr Treat Options Neurol 14, 230-240, doi:10.1007/s1 1940-012-0175-8 (2012).

39 Ratan, R. R. in Cell Death and Diseases of the Nervous System (ed V.E. Koliatsos, Ratan, R. R.) (Humana Press, 1999).

40 Polman, C. H. et al. Diagnostic criteria for multiple sclerosis: 2010 revisions to the McDonald criteria. Ann Neurol 69, 292-302, doi:10.1002/ana.22366 (2011).

41 Dhaeze, T. et al. CD70 defines a subset of proinflammatory and CNS-pathogenic TH1/TH17 lymphocytes and is overexpressed in multiple sclerosis. Cell Mol Immunol 16, 652-665, doi:10.1038/s41423-018-0198-5 (2019).

42 Kuhlmann, T. et al. An updated histological classification system for multiple sclerosis lesions. Acta Neuropathol 133, 13-24, doi:10.1007/s00401-016-1653-y (2017).

43 Dong, X. et al. Enhancers active in dopamine neurons are a primary link between genetic variation and neuropsychiatric disease. Nat Neurosci 21, 1482-1492, doi:10.1038/s41593018-0223-0 (2018).

44 Spratt, D. E. et al. A molecular explanation for the recessive nature of parkin-linked Parkinson's disease. Nat Commun 4, 1983, doi:10.1038/ncomms2983 (2013).

45 Kumar, A. et al. Disruption of the autoinhibited state primes the E3 ligase parkin for activation and catalysis. EMBO J 34, 2506-2521, doi:10.15252/embj.201592337 (2015).

46 Aguirre, J. D., Dunkerley, K. M., Mercier, P. \& Shaw, G. S. Structure of phosphorylated UBL domain and insights into PINK1-orchestrated parkin activation. Proc Natl Acad Sci U S A 114, 298-303, doi:10.1073/pnas.1613040114 (2017).

47 Kiss, R. et al. Structural features of human DJ-1 in distinct Cys 106 oxidative states and their relevance to its loss of function in disease. Biochim Biophys Acta Gen Subj 1861, 2619-2629, doi:10.1016/j.bbagen.2017.08.017 (2017).

48 Solti, K., Kuan, W. L., Fórizs, B., Kustos, G., Judith, M., Varga, Z., Herberth, B., Moravcsik, E., Kiss, R., Kárpáti, M., Mikes, A., Zhao, Y., Imre, T., Rochet, J.C., Aigbirhio, F., Williams-Gray, C. H., Barker, R. A., Tóth, G. DJ-1 can form beta-sheet structured aggregates that co-localize with pathological amyloid deposits. Neurobiology of Disease (2019).

49 Shevchenko, A., Tomas, H., Havlis, J., Olsen, J. V. \& Mann, M. In-gel digestion for mass spectrometric characterization of proteins and proteomes. Nat Protoc 1, 2856-2860, doi:10.1038/nprot.2006.468 (2006).

50 Shutinoski, B. et al. Lrrk2 alleles modulate inflammation during microbial infection of mice in a sex-dependent manner. Sci Transl Med 11, doi:10.1126/scitranslmed.aas9292 (2019). 
51 Yokochi, M., Narabayashi, H., Iizuka, R., \& Nagatsu T. Juvenile parkinsonism - some clinical, pharmacological, and neuropathological aspects. Adv Neurol 40, 407-413 (1984)

52 Gouider-Khouja, N., Larnaout, A., Amouri, R., et al. Autosomal recessive parkinsonism linked to parkin gene in a Tunisian family. Clinical, genetic and pathological study. Parkinsonism Relat Disord 9, 247-251.(2003) 

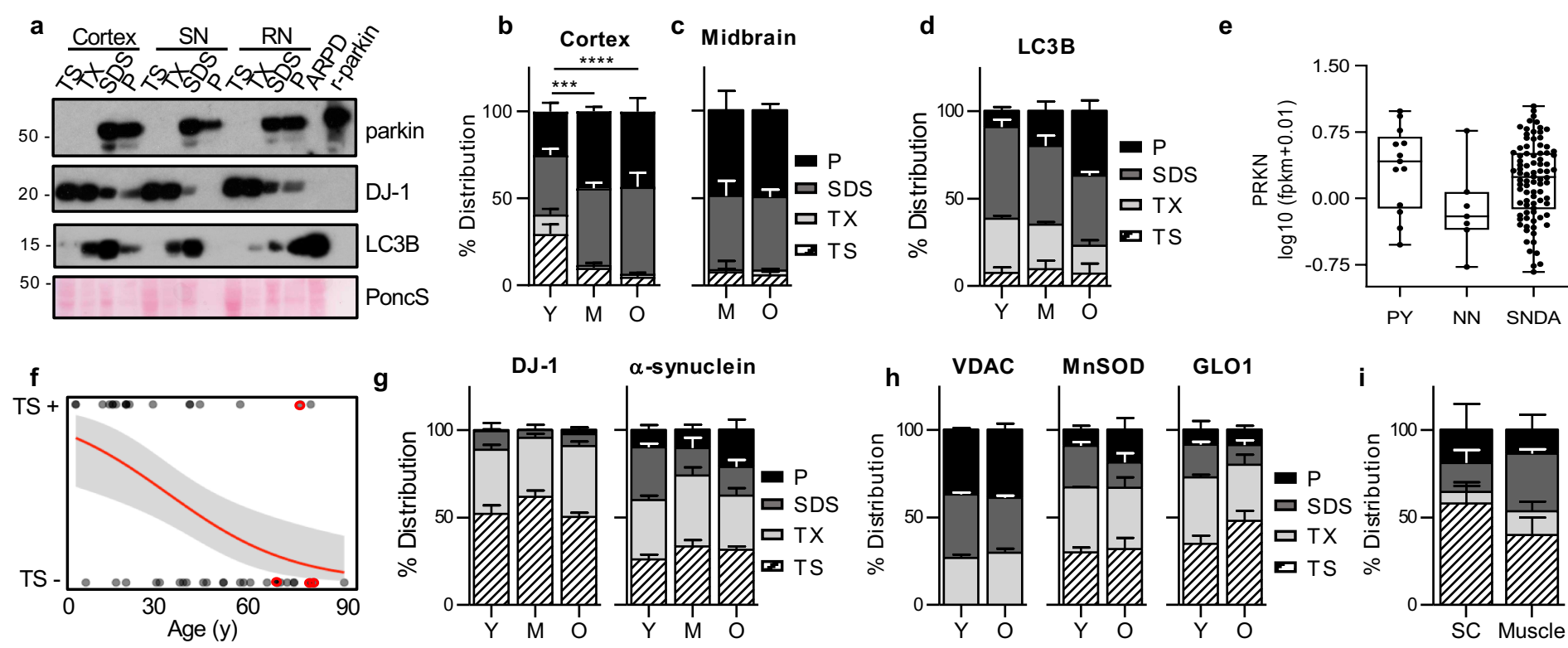
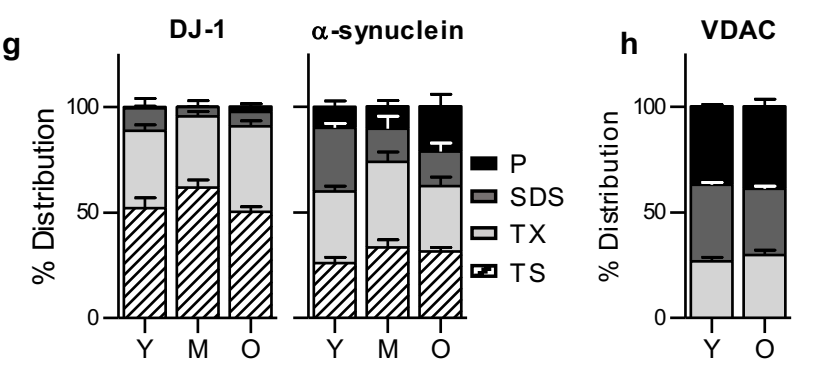

k

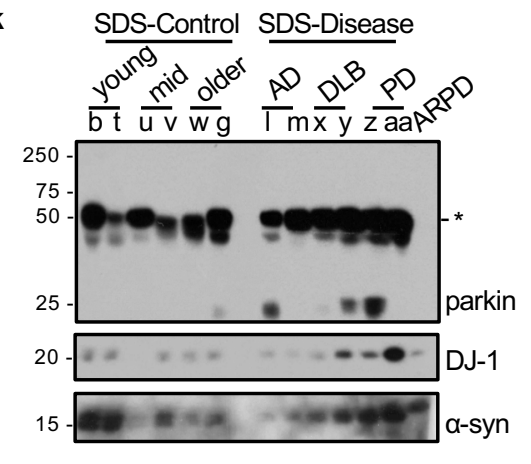

I
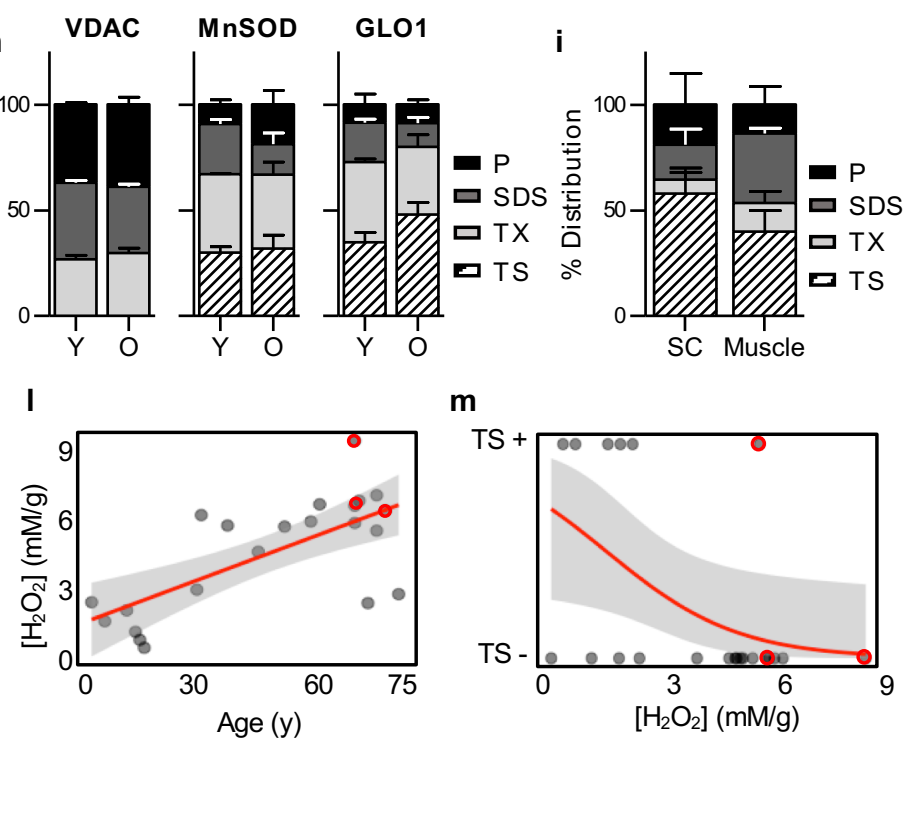

m

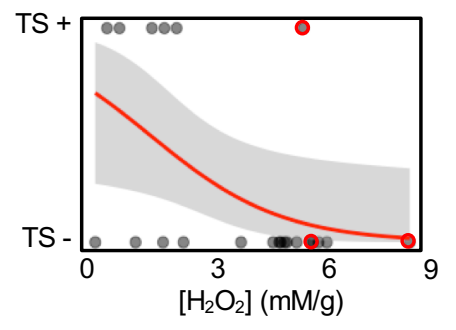

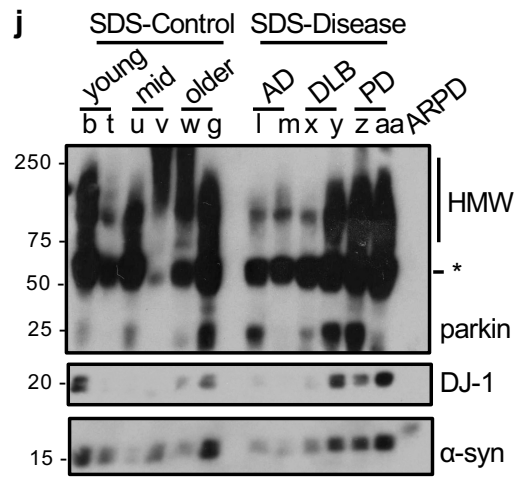

Figure 1: Parkin transitions from a soluble to an aggregated state in adult human midbrain.

(a) Representative Western blots of parkin, DJ-1, and LC3B distribution in human cortex, S. nigra (SN) and red nucleus (RN) brain specimens that had been serially fractionated into Tris-NaCl buffer-soluble (TS), Triton X-100-soluble (TX), 2\% SDS-soluble (SDS) extracts and the pellet (P) lysed in 30\% SDS-containing buffer. Lysates from PRKN-linked Parkinson disease (ARPD) brain and recombinant, human parkin (r-parkin) are included.

(b-c) Relative distribution of parkin signal within each fraction for (b) cortex and (c) midbrain grouped by age ranges: young (Y; $\leq 20 \mathrm{y} ; \mathrm{n}=13$ ); mid (M, >20y, <50y; $\mathrm{n}=15$ cortex, 6 midbrain); older (O, >50y; $\mathrm{n}=13$ cortex, 14 midbrain). Data shown as mean \pm SEM. Significance in protein distribution between soluble (TS+TX) and insoluble (SDS+pellet) fractions was determined using 2-way ANOVA ( $* * * p<0.001 ; * * * * p<0.0001)$. Additional Western blots in Extended Data Fig. 1a-c.

(d) Signal distribution for LC3B in human cortices as in (b). ( $n=3-5$ per age group).

(e) Quantification of log-transformed PRKN mRNA signals from individual pyramidal neurons (PY), non-neuronal leukocytes (NN) and S. nigra dopamine neurons (SNDA) isolated from healthy controls (ages, 59-89y).

(f) Logistic regression analysis of parkin solubility in cortices as a function of age $(n=45)$. Each brain is represented by an individual dot; red dots denote cases of late-onset Parkinson's not linked to $P R K N$; the logistic regression line (in red) and $95 \%$ confidence intervals (grey) are shown.

(g-h) Relative distribution of DJ-1, $\alpha$-synuclein (g) and (h) VDAC, MnSOD and GLO1 as described in (b). Representative Western blots in Extended Data Fig. 1b,c.

(i) Relative distribution of parkin as in (b) for human spinal cord $(n=4)$ and skeletal muscle specimens $(n=6)$, from donors aged 50-71y. Representative Western blots are in Extended Data Fig. 1e.

(j-k) Western blots of parkin, DJ-1, and $\alpha$-synuclein in SDS cortical fractions from healthy controls (3 age groups) and 6 patients including Parkinson's (PD), Alzheimer disease (AD) and dementia with Lewy bodies (DLB). The same lysates were separated by SDS-PAGE under (j) non-reducing and $(\mathbf{k})$ reducing conditions.

(I-m) Linear regression analyses of (l) $\mathrm{H}_{2} \mathrm{O}_{2}$ concentrations in control cortices (mM/g tissue) as a function of age for control cortices, and (m) logistic regression analyses of parkin solubility as a function of $\mathrm{H}_{2} \mathrm{O}_{2}$ levels in the same specimens $(n=20)$ as in (f). Red circles denote three disease cortices (AD; DLB; PD). 

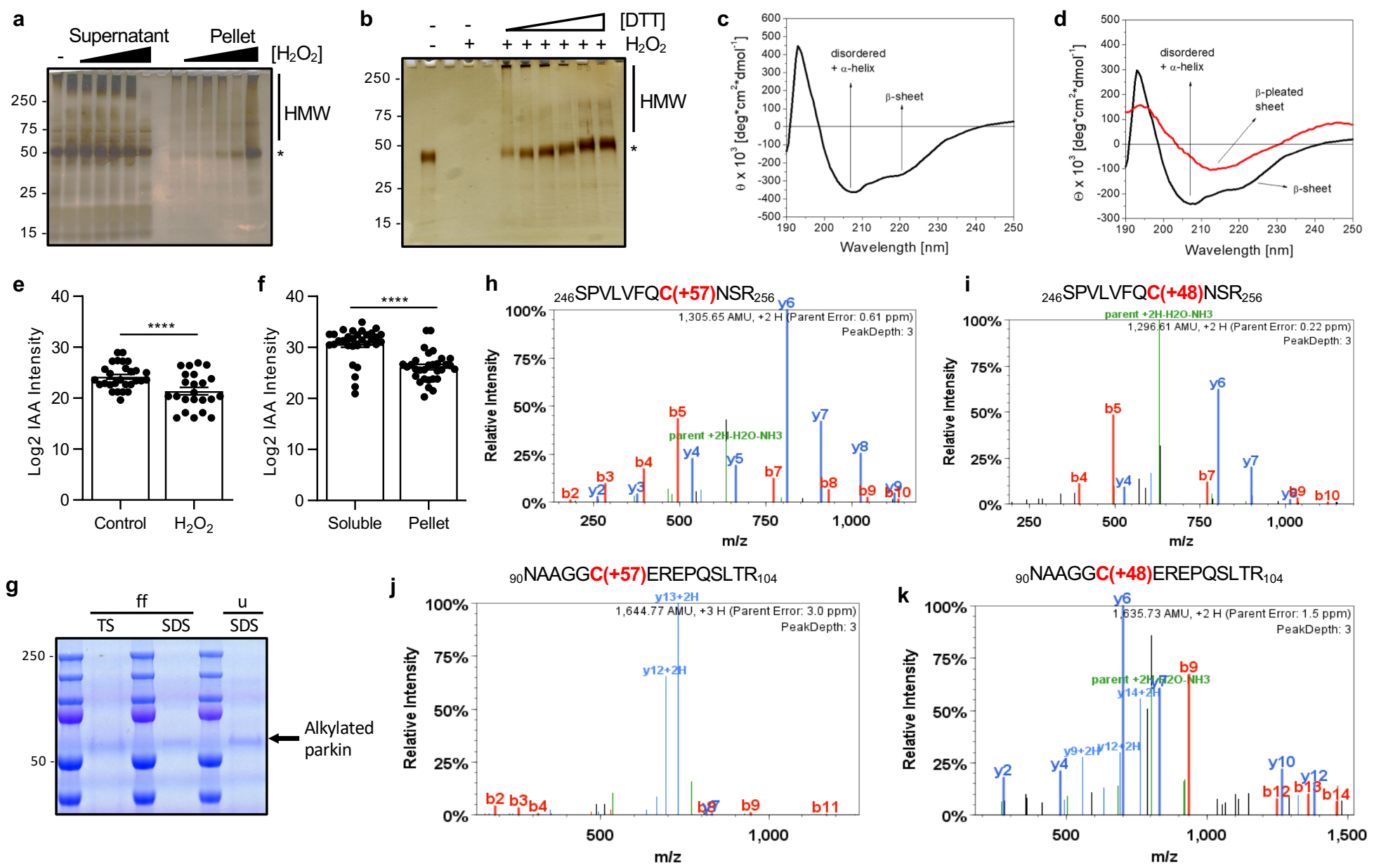

Figure 2: Parkin's secondary structure is altered by redox stress.

(a) Silver staining of r-parkin in soluble (supernatant) and insoluble (pellet) phases following exposure to increasing concentrations of $\mathrm{H}_{2} \mathrm{O}_{2}(0-2 \mathrm{mM})$ and run under non-reducing conditions. Monomer (*) and high $M_{r}$ weight $(\mathrm{HMW})$ r-parkin species are indicated.

(b) Silver stained gel of r-parkin exposed to $\mathrm{H}_{2} \mathrm{O}_{2}(10 \mathrm{mM})$, followed by treatment with increasing concentrations of DTT (0-100 $\mathrm{mM})$ prior to centrifugation and loading of the supernatant onto SDS-PAGE.

(c,d) Circular dichroism spectra of soluble, untreated r-parkin at (c) $\mathrm{T}=0$ and (d) soluble (black line) and aggregated (red line) states following incubation at $37^{\circ} \mathrm{C}$ for $\mathrm{T}=5$ days. The protein secondary structure shifts from a predominant appearance of $\alpha$-helix dominated state, as demonstrated by the positive band at $193 \mathrm{~nm}$ and negative bands $208 \mathrm{~nm}$ (black lines), to the appearance of $\beta$-pleated sheet formation, as demonstrated by negative bands at $218 \mathrm{~nm}$ and a rise in molar ellipticity with positive bands at $195 \mathrm{~nm}$ (red lines) during spontaneous oxidation.

(e-f) Quantitative analyses of IAA-modified cysteines captured by LC-MS/MS for (e) untreated $v s . \mathrm{H}_{2} \mathrm{O}_{2}$-exposed r-parkin, and (f) soluble compared to insoluble (pellet) fractions. Each data point represents the log2-transformed total IAA-signal intensities of single cysteine residues ( $\mathrm{n}=3$ runs for each). The cysteine pool is shown with the mean \pm SEM; significance $* * \mathrm{p}<0.01$ as determined using Student T-Test.

(g-k) LC-MS/MS-generated spectra following trypsin digestion of IAA alkylated WT parkin enriched from human cortex (example shown in (g)). (h-i) C253 (peptide aa246-256) was detected in both (h) a reduced state (IAA labelled; carbamidomethylation, +57 mass shift; peptide score 172.99) and (i) an oxidized state (i.e, trioxidation, +48 mass shift; peptide score 79.04). (j-k) C95 (peptide aa90-104) was also found in both a (j) reduced state (IAA labelled; carbamido-methylation, +57 mass shift; peptide score 100.16) and (k) oxidized state (trioxidation, +48 mass shift; peptide score 84.47). See also Extended Data Table 1 (human cortex) and Extended Data Fig. 4h (r-parkin). 

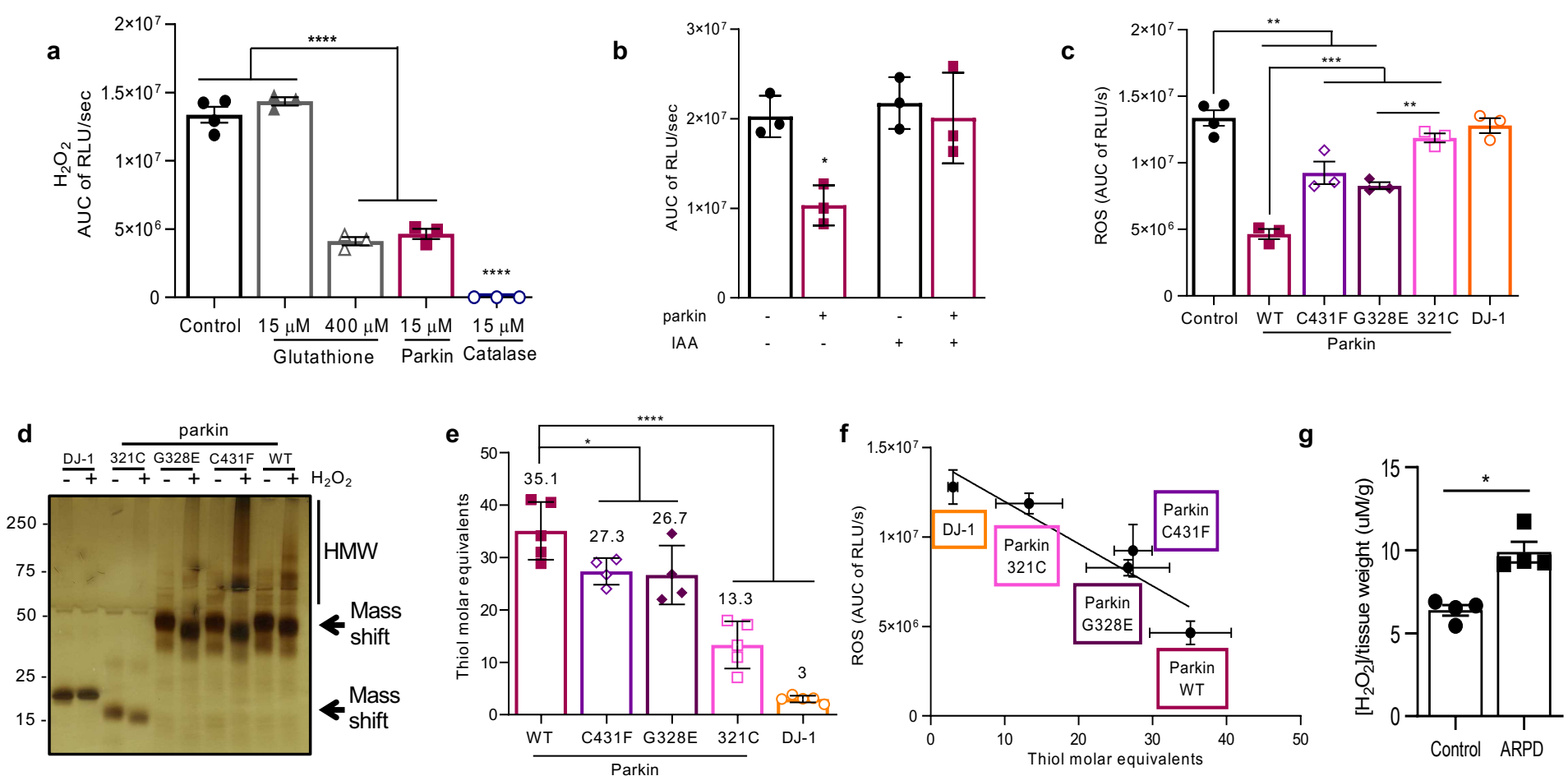

Figure 3: Parkin reduces $\mathrm{H}_{2} \mathrm{O}_{2}$ to water.

(a-d) Area under the curve (AUC) plots for results from in vitro colorimetric $\mathrm{H}_{2} \mathrm{O}_{2}$ assays, where AUC integrates total $\mathrm{H}_{2} \mathrm{O}_{2}$ present over the time course of the assay (see also Extended Data Fig. 5a). (a) WT full-length r-parkin and glutathione significantly reduced $\mathrm{H}_{2} \mathrm{O}_{2}$; (b) This was blocked when r-parkin was pre-incubated with IAA. (c) Comparison of WT rparkin with DJ-1, r-parkin point mutants, and r-parkin ${ }_{321-465}$ (321C). Results represent an average of $\mathrm{n}=3 \pm \mathrm{SD} ;{ }^{*} \mathrm{p}<0.05$, $* * \mathrm{p}<0.01,{ }^{* * *} \mathrm{p}<0.001$, and $* * * * \mathrm{p}<0.0001$ using one-way ANOVA with Tukey's post hoc test. (d) Visualization of proteins by silver staining (non-reducing conditions).

(e) Quantification of reactive thiol content (in molar equivalents) of r-parkin WT, r-parkin point mutants, r-parkin $321-465$ and full-length r-DJ-1 using the Ellman's reagent assay.

(f) Correlation curve between number of free thiols (e) and $\mathrm{H}_{2} \mathrm{O}_{2}$ reducing capacity (c) for indicated proteins.

(g) Quantification of $\mathrm{H}_{2} \mathrm{O}_{2}$ in human brain from PRKN-deficient ARPD cortices compared to age- and post-mortem intervalmatched controls collected at the same institution. Results are represented as the mean concentration of $\mathrm{H}_{2} \mathrm{O}_{2}$ per $g$ of tissue analyzed $(\mu \mathrm{M} / \mathrm{g} ; \mathrm{n}=4$ /group $) \pm \mathrm{SEM} ;{ }^{*} \mathrm{p}<0.05$ determined using Student T-test. 

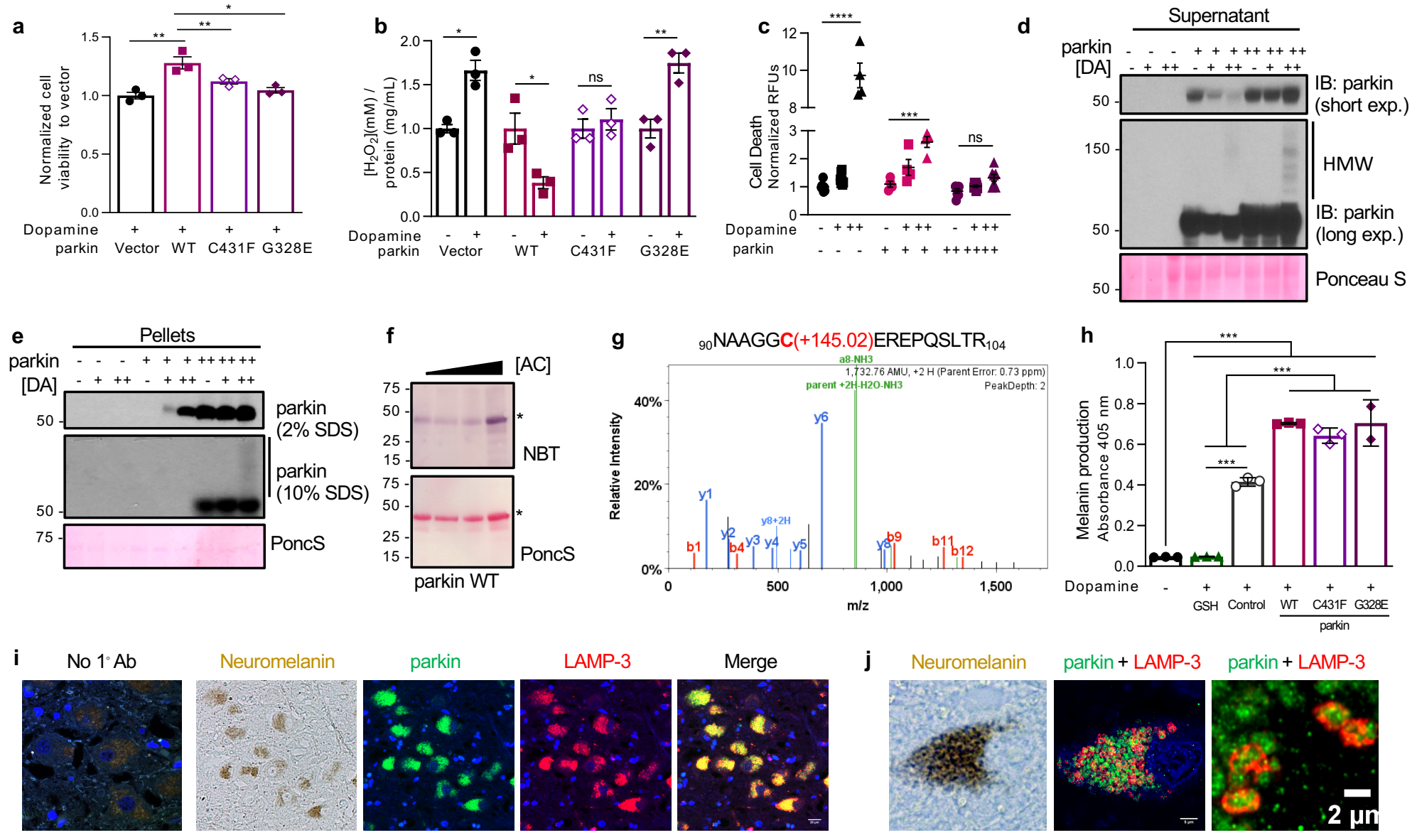

Figure 4: Parkin directly conjugates dopamine metabolites and is associated with neuromelanin in vivo.

(a-b) Cell viability assay (a) and (b) $\mathrm{H}_{2} \mathrm{O}_{2}$ quantification for dopamine-treated M17 cells expressing WT or two ARPDlinked parkin mutants. Cells were exposed to $200 \mu \mathrm{M}$ dopamine or vehicle for $20 \mathrm{~h}$ as indicated. Data represent the mean of duplicates \pm SEM. ${ }^{*} \mathrm{p}<0.05$ by 1 -way ANOVA and is representative of $\mathrm{n}=3$.

(c) Cell viability assay of dopamine-stressed human M17 neuroblastoma cells that stably express vector-control plasmid (parkin -) or human PRKN cDNA at mid- (+) or high (++) levels. Cells were stressed with $20 \mu \mathrm{M}(+)$ and $200 \mu \mathrm{M}(++)$ dopamine for $20 \mathrm{~h}$. Representative data are shown for the mean of duplicates \pm SEM from $n=4-8$ independent experiments (for different concentrations); ${ }^{*} \mathrm{p}<0.05$ by 1 -way ANOVA.

(d-e) Western blot of parkin in soluble (supernatant, d) and insoluble (serial pellets, e) fractions of lysates described in (c). SDS/PAGE was run under reducing conditions.

(f) NBT and Ponceau S staining of WT human r-parkin that had been incubated with increasing concentrations of aminochrome in vitro; gel was run under reducing conditions. *Denotes parkin monomers.

(g) LC-MS/MS-generated spectra highlighting trypsin-digested human r-parkin peptide aa90-104 carrying a indole-5,6quinone adduct (+145 mass gain) at C95 (Mascot ion score 31.1) See also Extended Data Fig. 6c,d.

(h) AUC of kinetic result of in vitro melanin formation as monitored by a colorimetric assay. Dopamine polymerization to melanin was monitored in the presence or absence (blank) of WT, r-parkin point mutants and glutathione (GSH). Data represent the mean of triplicates \pm SEM. $* * * p<0.05$ by 1-way ANOVA. See also Extended Data Fig. 6b.

(i-j) Indirect immunofluorescence microscopy signals generated by anti-parkin (clone-E; green colour) identifies perinuclear organelles throughout the neuronal cytoplasm that are also recognized by anti-LAMP-3/CD63 (red colour), as shown by double-labelling (blue colour, Hoechst stain). Reactivities generated by anti-parkin topographically overlap with juxtanuclear neuromelanin, as shown by panel to the left identifying neuromelanin-carrying cells by light microscopy in the same field. No primary Ab control run in parallel to experiment is shown. Higher magnification of individual dopaminergic neuron is shown in (j). 

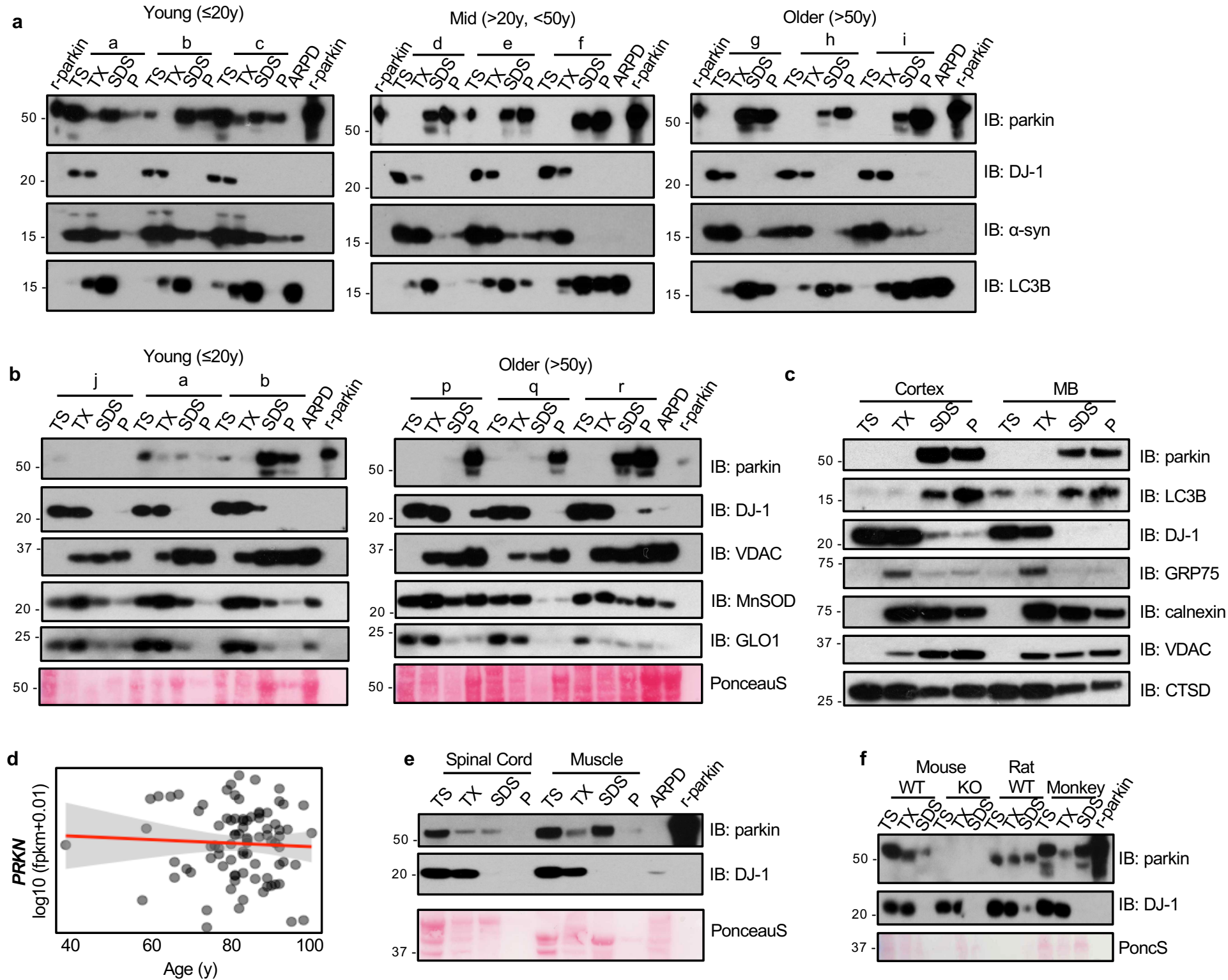

Extended Data Figure 1. Parkin transitions from a soluble to an aggregated state in adult human brain.

(a) Western blots of parkin, DJ-1, $\alpha$-synuclein and LC3B distribution in 9 representative human cortex samples identified by lowercase letter code (see Supplementary Information Table 1). Tissue fractionation and age ranges were as described in Fig. 1; gels were run under reducing conditions. ARPD lysates and r-parkin are included as controls.

(b) Western blots of parkin, DJ-1, VDAC, MnSOD and glyoxylase-1 proteins, and Ponceau S staining in serially fractionated human cortices from younger $(n=3)$ and older $(n=3)$ individuals. Quantification of relative protein distribution is shown in Fig. $1 \mathrm{~h}$.

(c) Western blot of indicated proteins from serially fractionated cortex and midbrain tissue as described in (a).

(d) Linear regression analysis of log transformed $P R K N$ transcripts as a function of age in human control S. nigra dopamine neurons where each dot represents values for a single neuron as shown in Fig. 1e.

(e) Western blot of parkin and DJ-1 expression in serial fractions from representative human spinal cord and skeletal muscle tissues from individuals $\geq 50 \mathrm{y}$. Relative protein distribution is shown in Fig. 1i.

(f) Western blot of parkin and DJ-1 and Ponceau S staining of serial fractions from whole brains of wild-type (WT; 8 mths of age) and Prkn knock-out (KO) mice, rat (WT; $14 \mathrm{mths}$ ) and cortex from cynomolgus monkey (60 mths). 
a

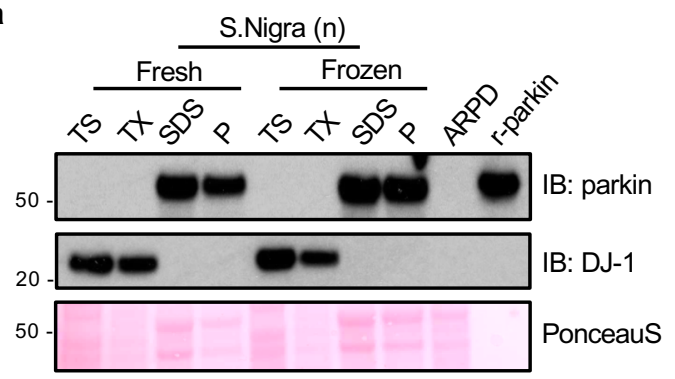

d

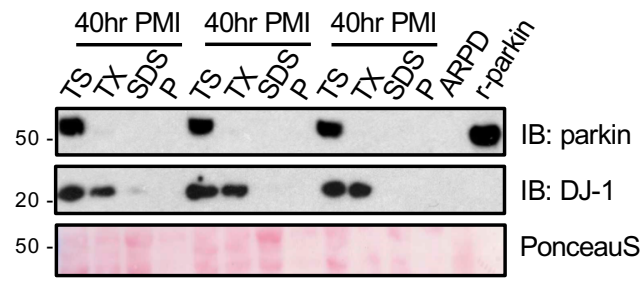

b

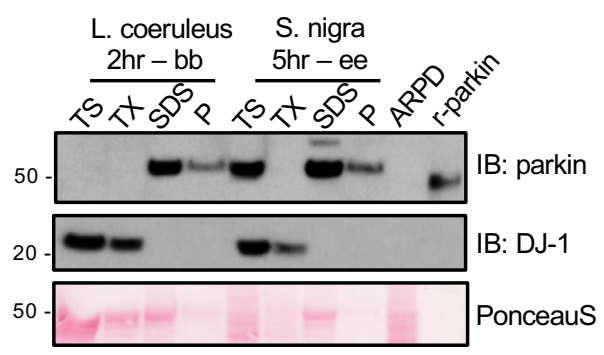

e $\frac{\mathrm{p}}{\frac{\mathrm{pH} 5.5}{5} \frac{\mathrm{pH} 7.0}{5}} \frac{\mathrm{pH} 5.5}{\mathrm{pH} 7.0}$

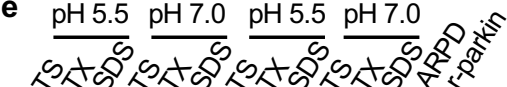
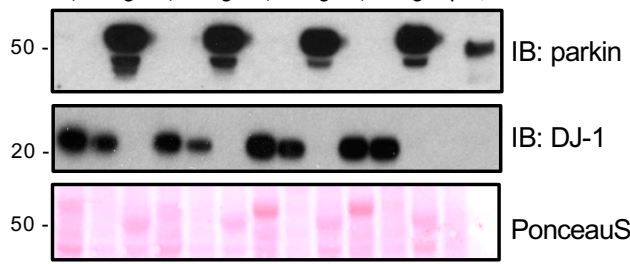

C

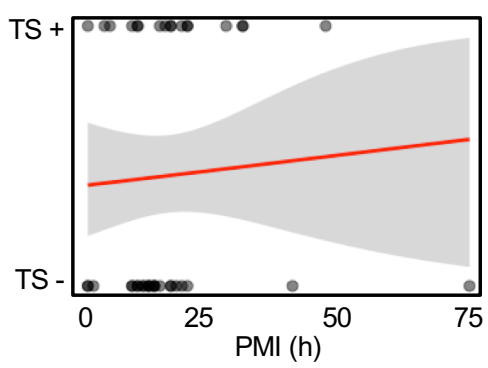

\section{Extended Data Figure 2. Parkin solubility is not affected by tissue freezing, length of post mortem interval or pH changes.}

(a-b) Western blot of parkin and DJ-1 distribution of (a) human S. nigra (brain code n) lysates that had been processed in parallel with $v s$. without a freeze/thaw step prior to serial fractionation, and (b) from L. coeruleus and $S$. nigra which were collected within 2-5 hrs after death prior to freezing.

(c) Logistic regression analysis of parkin solubility as a function of length for post mortem interval (PMI; in hrs); the logistic regression analysis line (red) and 95\% confidence intervals (grey) are shown ( $\mathrm{n}=45$ cortices).

(d) Immunoblots for endogenous parkin and dj-1 signals from serially extracted, WT mouse brains $(\mathrm{n}=3)$ dissected following $40 \mathrm{hr}$ post mortem interval at $4^{\circ} \mathrm{C}$.

(e) Western blotting of parkin and DJ-1 distribution and Ponceau S staining for fractions of human cortices serially extracted in parallel using standard buffers with varying $\mathrm{pH}$, as indicated. 


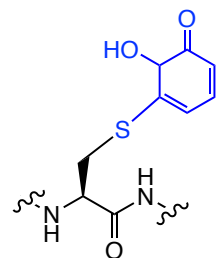

S-quinone adduct

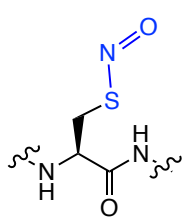

S-nitrosothiol

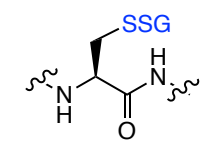

S-glutathione

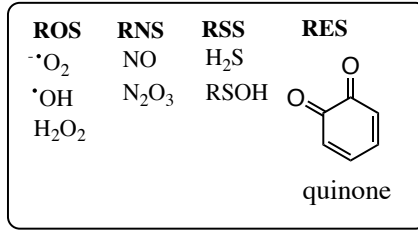

GSH

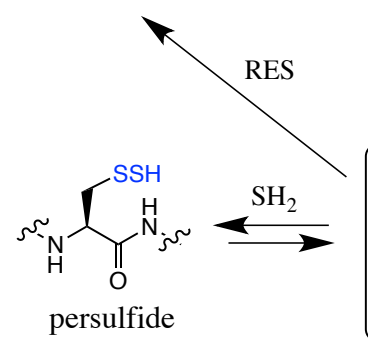

$$
\text { RNS } \uparrow
$$

GSSG

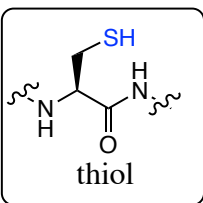

ROS/RNS

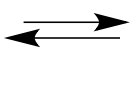

$\uparrow \downarrow$

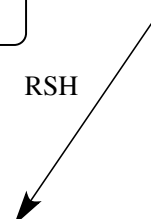

$\mathrm{ROS}+\mathrm{RSH}$

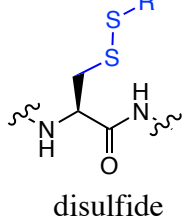

disulfide

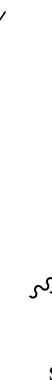<smiles>C1CCCCC1</smiles>

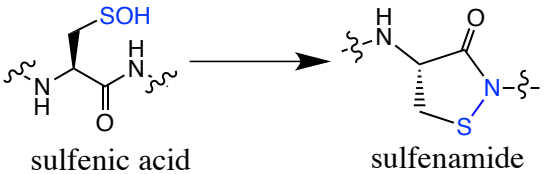
ROS/RNS

$\checkmark$

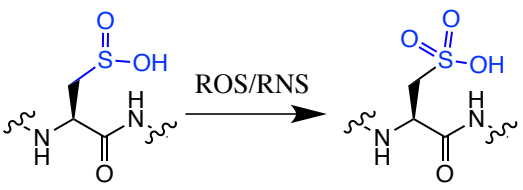

sulfinic acid

\section{Extended Data Figure 3. Summary of redox-related thiol chemistry.}

Schema of select, reversible and irreversible cysteine modifications that can occur on thiols due to attacks by reactive oxygen species (ROS), -nitrogen species (RNS), -sulfur species (RSS) and -electrophilic species (RES), which include quinones. This graphic summary was modified from Alcock et al., 2018. 
bioRxiv preprint doi: https://doi.org/10.1101/2020.02.19.953034; this version posted February 20, 2020. The copyright holder for this preprint (which was not certified by peer review) is the author/funder. All rights reserved. No reuse allowed without permission.
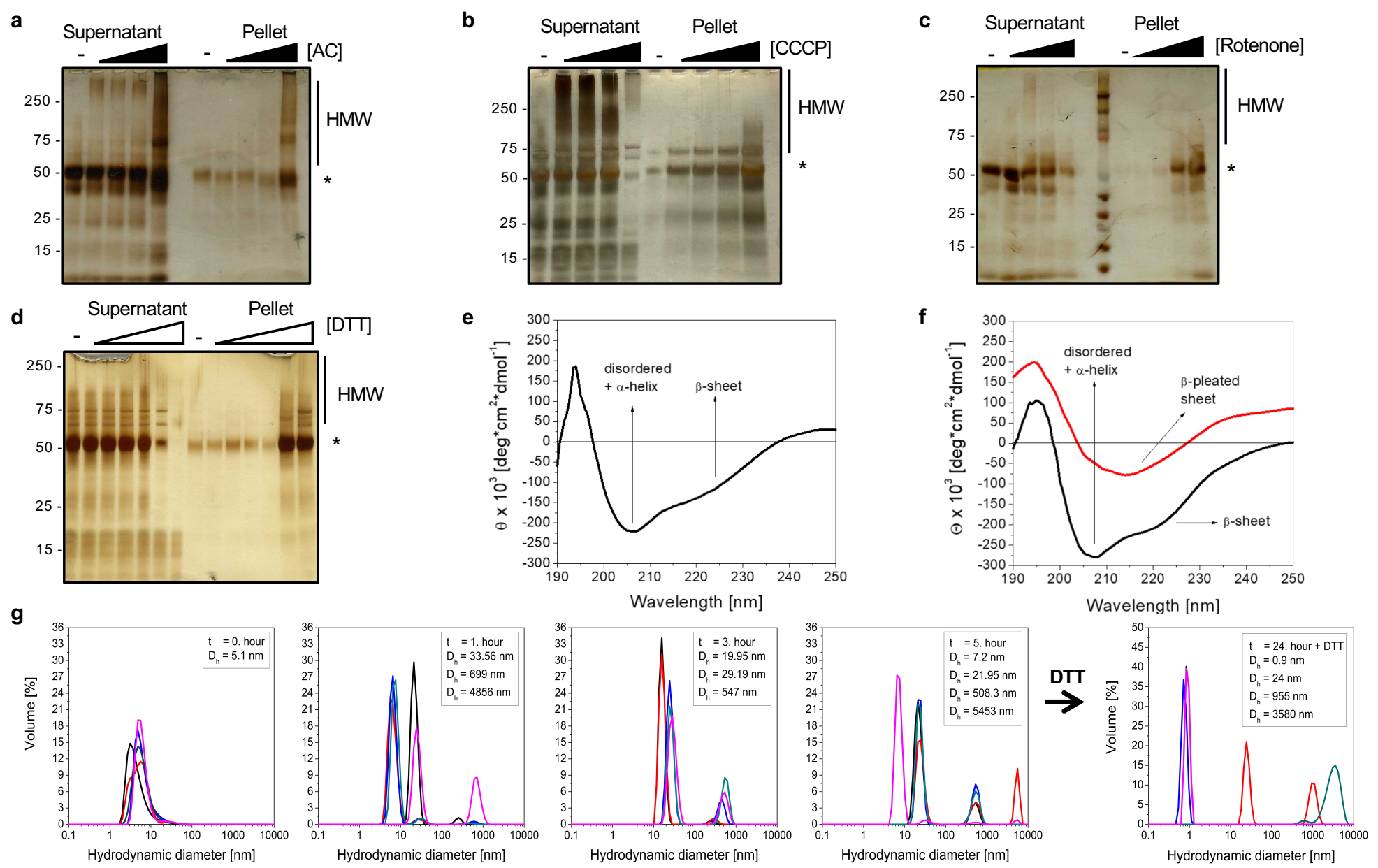

h

\begin{tabular}{|c|c|c|c|c|c|c|c|}
\hline Run & $\mathrm{IAA}+\mathrm{NEM}$ & IAA & $\mathrm{H}_{2} \mathrm{O}_{2} 20 \mu \mathrm{M}$ & $\mathrm{H}_{2} \mathrm{O}_{2} 1 \mathrm{mM}$ & $\mathrm{H}_{2} \mathrm{O}_{2} 4.5 \mathrm{mM}$ & $\mathrm{H}_{2} \mathrm{O}_{2} 4.5 \mathrm{mM}$ & $\mathrm{H}_{2} \mathrm{O}_{2} 4.5 \mathrm{mM}$ \\
\hline $\begin{array}{c}\text { \% Parkin protein } \\
\text { coverage }\end{array}$ & 83 & 89 & 57 & 60 & 86 & 98 & 97 \\
\hline \# peptides identified & 40 & 35 & 22 & 33 & 38 & 51 & 47 \\
\hline \# Cysteines identified & 27 & 31 & 16 & 21 & 28 & 34 & 34 \\
\hline \# IAA-cysteines & 27 & 30 & 16 & 21 & 28 & 34 & 34 \\
\hline $\begin{array}{c}\text { IAA-cys/ } \\
\text { identified-cys }\end{array}$ & $27 / 27$ & $30 / 31$ & $16 / 16$ & $21 / 21$ & $28 / 28$ & $34 / 34$ & $34 / 34$ \\
\hline$\%$ & 100 & 97 & 100 & 100 & 100 & 100 & 100 \\
\hline \# NEM-cysteines & 0 & $\mathrm{n} / \mathrm{a}$ & 3 & 5 & 26 & 16 & 14 \\
\hline $\begin{array}{l}\text { NEM-cys/ } \\
\text { identified-cys }\end{array}$ & $0 / 27$ & $\mathrm{n} / \mathrm{a}$ & $3 / 16$ & $5 / 21$ & $26 / 28$ & $16 / 34$ & $14 / 34$ \\
\hline$\%$ & 0 & $\mathrm{n} / \mathrm{a}$ & 19 & 24 & 93 & 47 & 41 \\
\hline
\end{tabular}

\section{Extended Data Figure 4. Oxidation of parkin thiols promotes insolubility.}

(a-d) Detection of r-parkin in soluble (supernatant) and insoluble phases (pellet; recovered by $10 \%$ SDS-containing buffer) of following exposure to increasing concentrations of (a) aminochrome $(0-200 \mu \mathrm{M}),(\mathbf{b})$ CCCP $(0-200 \mu \mathrm{M}),(\mathbf{c})$ rotenone $(0$ $2 \mathrm{mM})$ and (d) DTT (0-1M) as described in Fig. 2a.

(e-f) Circular dichroism spectra of soluble, $\mathrm{H}_{2} \mathrm{O}_{2}$-exposed WT r-parkin secondary structure at (e) $\mathrm{T}=0$ and (f) soluble (black line) and aggregated (red line) states following incubation at $37^{\circ} \mathrm{C}$ for $\mathrm{T}=5$ days to promote spontaneous oxidation as described in Fig. 2c,d.

(g) Dynamic light scattering analysis showing progressive size changes, as measured in hydrodynamic diameters (nm), was monitored during $5 \mathrm{hrs}$ at room temperature. The structural state for wild-type, r-parkin under non-reducing, native conditions showed increased aggregate formation over time, which was partially reversed by treatment with DTT as indicated.

(h) Summary of LC-MS/MS based analysis of cysteine oxidation state of untreated and $\mathrm{H}_{2} \mathrm{O}_{2}$-treated r-parkin as indicated using IAA-DTT-NEM fingerprinting to identify reduced cysteines (IAA) or reversibly-oxidized residues (NEM). For comparison, see Extended Data Table 1 that lists modified cysteines identified in parkin purified from human brain cortex. A complete list of modified cysteines under each condition tested is found in Supplemental Information Table 2. 
a
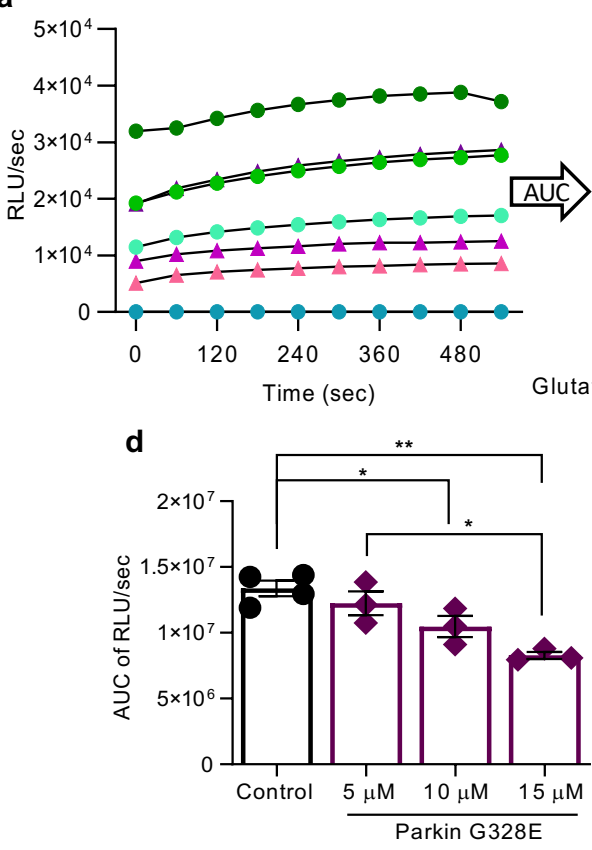

b

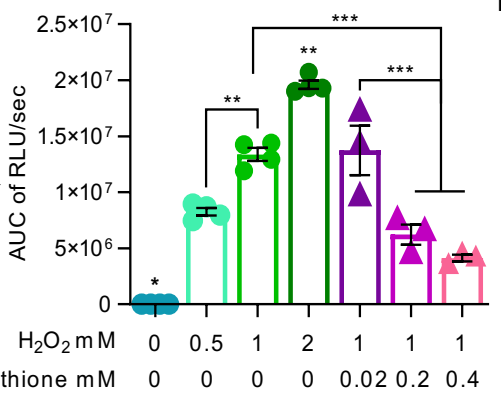

e

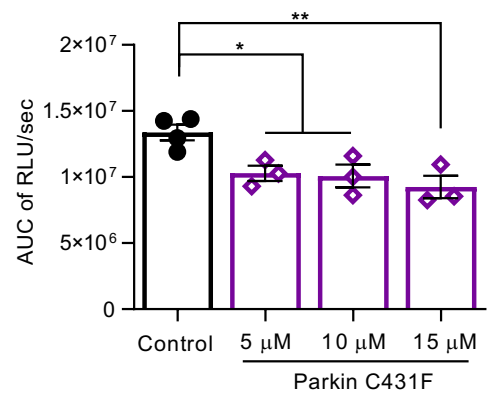

C

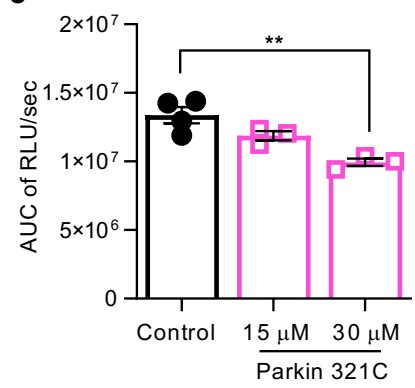

f

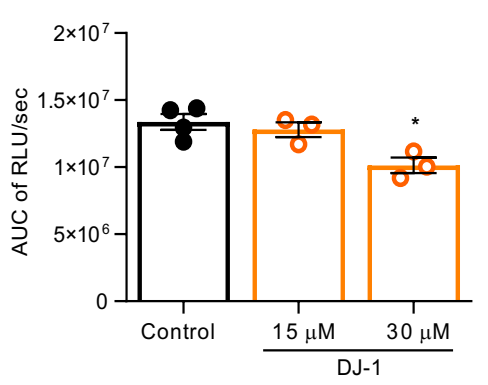

Extended Data Figure 5. Parkin directly reduces $\mathrm{H}_{2} \mathrm{O}_{2}$ to water in a concentration-dependent manner.

(a) Kinetic readings from in vitro colorimetric $\mathrm{H}_{2} \mathrm{O}_{2}$ assays comparing increasing concentrations of input $\mathrm{H} 2 \mathrm{O} 2$ and the effect of increasing concentrations of glutathione. Curves were then converted to the area under the curve (AUC) where AUC integrates the total value of $\mathrm{H}_{2} \mathrm{O}_{2}$ signals generated over the 10 minutes time course of the assay.

(b-f) AUC graphs for results from in vitro $\mathrm{H}_{2} \mathrm{O}_{2}$ assays for various concentrations of recombinant proteins, as indicated. 

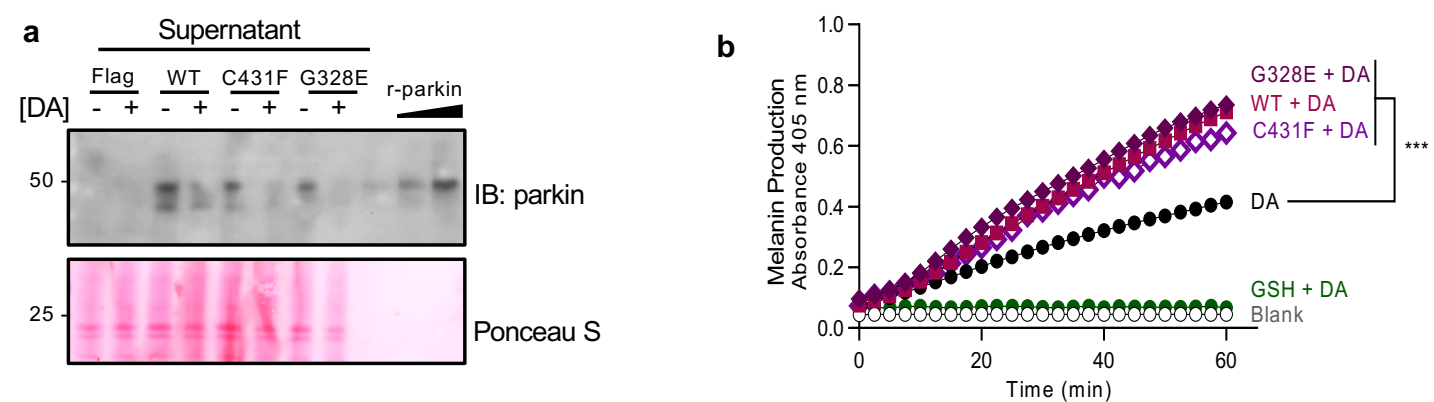

C

\begin{tabular}{|c|c|c|c|c|c|c|c|}
\hline Peptide sequence & $\begin{array}{c}\text { Peptide } \\
\text { start } \\
\text { index }\end{array}$ & $\begin{array}{l}\text { Peptide } \\
\text { stop } \\
\text { index }\end{array}$ & $\begin{array}{l}\text { Variable modifications identified by } \\
\text { spectrum }\end{array}$ & Observed $\mathrm{m} / \mathrm{z}$ & $\begin{array}{l}\text { Spectrum } \\
\text { charge }\end{array}$ & $\begin{array}{l}\text { Actual } \\
\text { peptide } \\
\text { mass } \\
(\mathrm{AMU}) \\
\end{array}$ & $\begin{array}{c}\text { Peptide } \\
\text { identification } \\
\text { probability }\end{array}$ \\
\hline NAAGGcEREPQSLTR & 90 & 104 & C95 Aminochrome (+147) & 579.265625 & 3 & $1,734.78$ & $98.00 \%$ \\
\hline NAAGGcEREPQSLTR & 90 & 104 & C95 Aminochrome $(+147)$ & 579.26532 & 3 & $1,734.77$ & $99.70 \%$ \\
\hline NAAGGcEREPQSLTR & 90 & 104 & C95 Aminochrome (+147) & 579.266418 & 3 & $1,734.78$ & $99.00 \%$ \\
\hline NAAGGcEREPQSLTR & 90 & 104 & C95 Aminochrome (+147) & 579.266663 & 3 & $1,734.78$ & $99.70 \%$ \\
\hline NAAGGcEREPQSLTR & 90 & 104 & C95 Dopamine $(+151)$ & 580.60907 & 3 & $1,738.81$ & $96.20 \%$ \\
\hline NAAGGcEREPQSLTR & 90 & 104 & C95 Dopamine (+151) & 580.609802 & 3 & $1,738.81$ & $95.50 \%$ \\
\hline NAAGGcEREPQSLTR & 90 & 104 & C95 Indole-5,6-quinone $(+145)$ & 578.593872 & 3 & $1,732.76$ & $99.50 \%$ \\
\hline NAAGGcEREPQSLTR & 90 & 104 & C95 Indole-5,6-quinone (+145) & 578.593994 & 3 & $1,732.76$ & $99.70 \%$ \\
\hline NAAGGcEREPQSLTR & 90 & 104 & C95 Indole-5,6-quinone $(+145)$ & 867.386719 & 2 & $1,732.76$ & $99.70 \%$ \\
\hline NAAGGcEREPQSLTR & 90 & 104 & C95 Indole-5,6-quinone $(+145)$ & 867.387329 & 2 & $1,732.76$ & $99.70 \%$ \\
\hline NAAGGcEREPQSLTR & 90 & 104 & C95 Indole-5,6-quinone $(+145)$ & 867.387512 & 2 & $1,732.76$ & $98.80 \%$ \\
\hline NAAGGCEREPQSLTR & 90 & 104 & $\begin{array}{l}\text { C95 Indole-5,6-quinone }(+145) \\
\text { M80 Oxidation }(+15.99), \text { C95 }\end{array}$ & 867.386292 & 2 & $1,732.76$ & $99.60 \%$ \\
\hline KGQEmNATGGDDPRNAAGGcEREPQSLTR & 76 & 104 & Aminochrome $(+147)$ & 803.108948 & 4 & $3,208.41$ & $99.70 \%$ \\
\hline KGQEmNATGGDDPRNAAGGcEREPQSLTR & 76 & 104 & $\begin{array}{l}0 \text { Oxidation (+15.99), C95 Dopamine } \\
\text { Quinone }(+151)\end{array}$ & 803.862549 & 4 & $3,211.42$ & $97.80 \%$ \\
\hline
\end{tabular}

d<smiles>O=CSC1=C2[NH2+]C=CC2=CC(=O)C1=O</smiles>

Indole-5,6-quinone $+\mathrm{C}_{8} \mathrm{O}_{2} \mathrm{NH}_{3}$

Mass shift $=+145$<smiles>[R]CSC1=C2NCCC2=CC(=O)C1=O</smiles>

$$
\begin{gathered}
\text { Aminochrome } \\
+\mathrm{C}_{8} \mathrm{O}_{2} \mathrm{NH}_{5} \\
\text { Mass shift }=+147
\end{gathered}
$$<smiles>[R]CSc1cc(CCN)cc(O)c1O</smiles>

$$
\begin{aligned}
& \text { Dopamine } \\
& +\mathrm{C}_{8} \mathrm{O}_{2} \mathrm{NH}_{9}
\end{aligned}
$$$$
\text { Mass shift }=+151
$$

\section{Extended Data Figure 6. Parkin conjugates dopamine metabolites at C95}

(a) Western blot analysis of ectopic, WT and mutant parkin expression in dopamine-treated M17 cells, as described in Fig. 4a,b.

(b) In vitro melanin formation, as monitored by a colorimetric assay (absorbance rate, $405 \mathrm{~nm}$ ) demonstrating increased polymerization of dopamine (DA) over a $60 \mathrm{~min}$ period in the presence or absence (blank) of wild-type (WT) r-parkin and two point mutants $(\mathrm{G} 328 \mathrm{E}$; C431F). Melanin synthesis from dopamine is blocked in the presence of glutathione (GSH). Data represent the mean of triplicates \pm SEM. $* * * p<0.05$ by 1 -way ANOVA.

(c) Table summarizing LC-MS/MS-based detection of adducts of dopamine-metabolites conjugated to C95 of r-parkin following exposure of r-parkin to aminochrome. Chemical structures for identified adducts are shown in (d). 


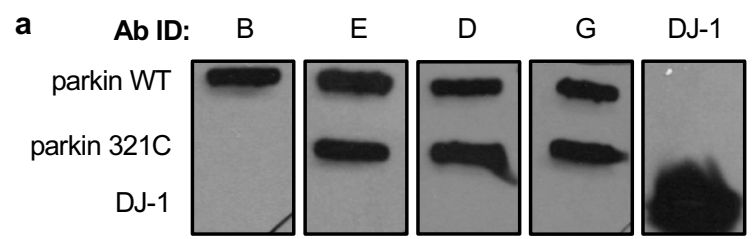

b

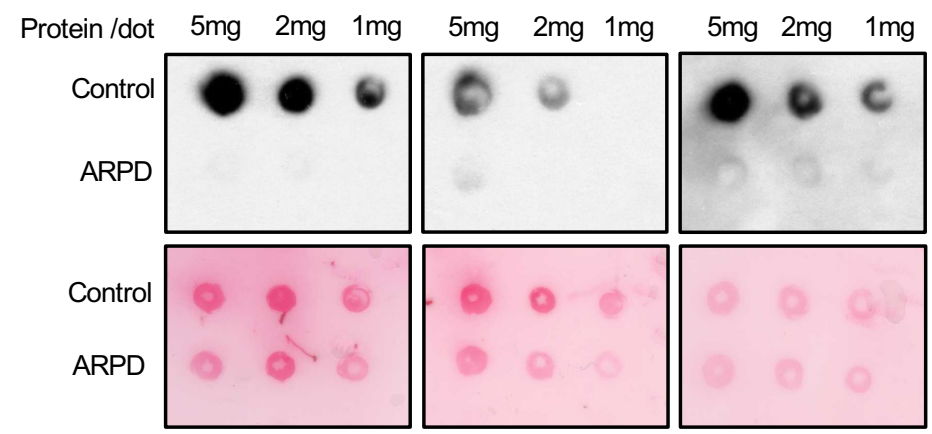

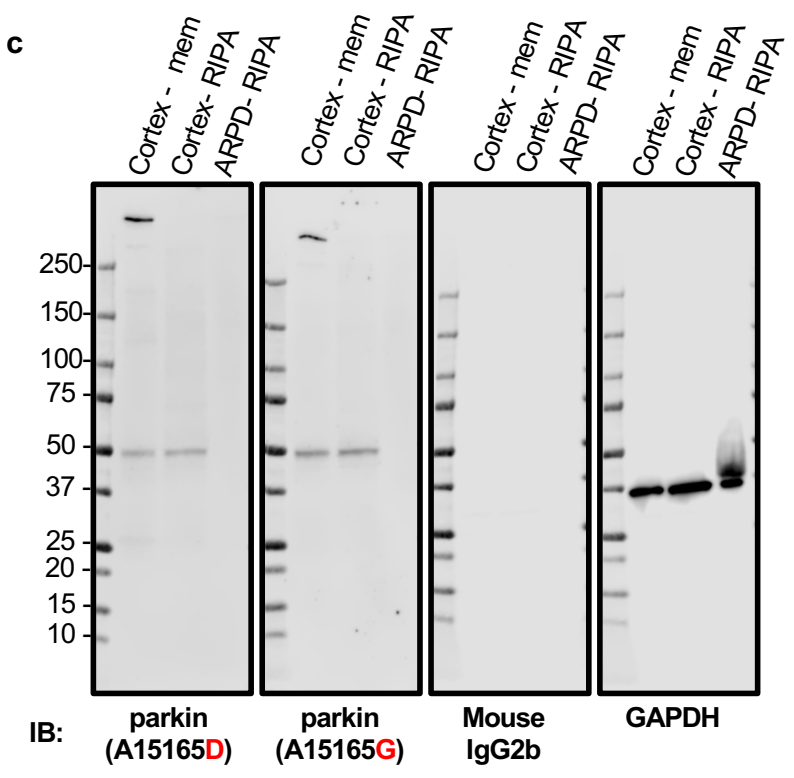

d

\begin{tabular}{|l|l|l|}
\hline Clone & Epitope & Domain \\
\hline A15165B & aa $89-93$ & Linker \\
\hline A15165D & aa $397-404$ & REP \\
\hline A15165G & aa $398-404$ & REP \\
\hline A15165E & aa $177-184 ;$ aa 443-453; aa 459-464 & RING0; RING; RING2 [conformational] \\
\hline
\end{tabular}

\section{Extended Data Figure 7. New monoclonal antibodies visualize parkin in ageing human midbrain.}

(a-c) Characterization of four murine, monoclonal antibodies (of $\mathrm{IgG}_{2}$ isotype; clone-B, -E, -D, and -G) in three different assays: (a) against recombinant (r-), full-length, untagged, wildtype (WT) human parkin using non-denaturing slot blots (100ng/slot) of original antigen as well as truncated r-parkin $321-465$ and full-length, untagged, human r-DJ-1; (b) against human brain lysates (SDS fractions from control and PRKN-linked ARPD cases) using non-denaturing dot blots; and (c) by denaturing SDS/PAGE and Western blotting of extracts from cortical specimens of a control brain and a parkin-deficient ARPD case. Screening by these three methods as well as by cell-based microscopy (using indirect immunofluorescence) revealed specific staining for four anti-parkin clones (-B, -E, -D and -G), which was conformation-dependent for clone-E. (d) List of epitopes within the sequence of human parkin, as recognized by clones -B, -E, -D, and -G and informed by overlapping screening with 7-12 amino acid-long peptides covering full-length, human parkin. Note that the clone E epitope is conformational, comprised of the three regions indicated. 
bioRxiv preprint doi: https://doi.org/10.1101/2020.02.19.953034; this version posted February 20, 2020. The copyright holder for this preprint
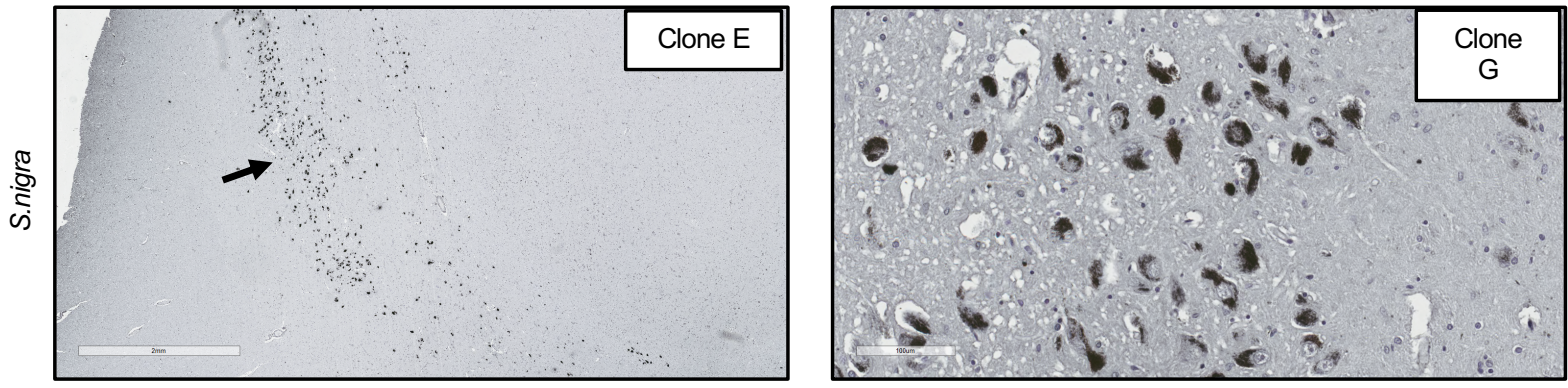

b
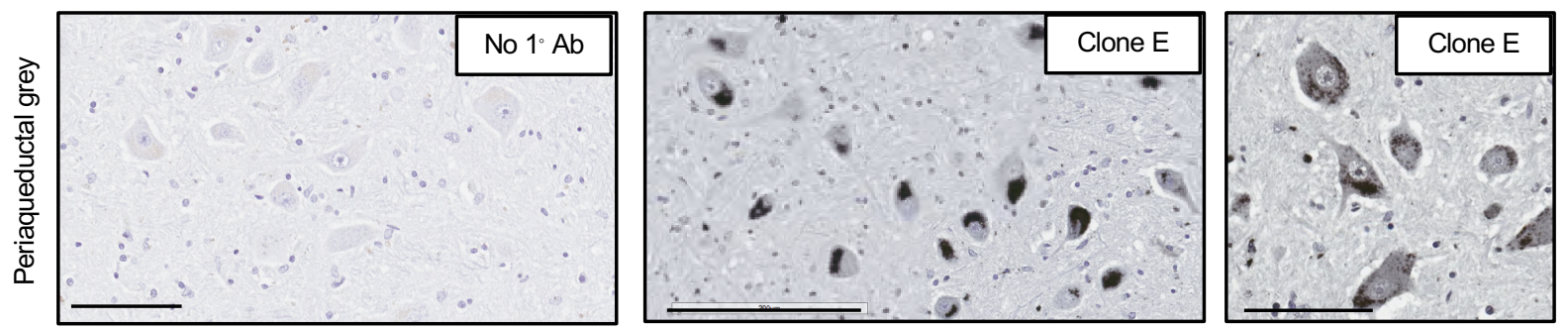

C
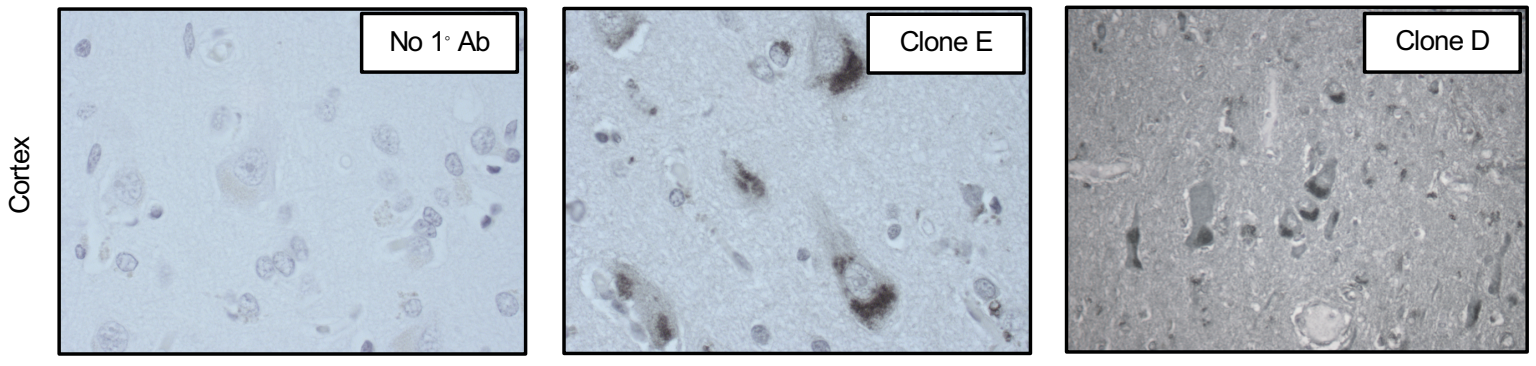

d
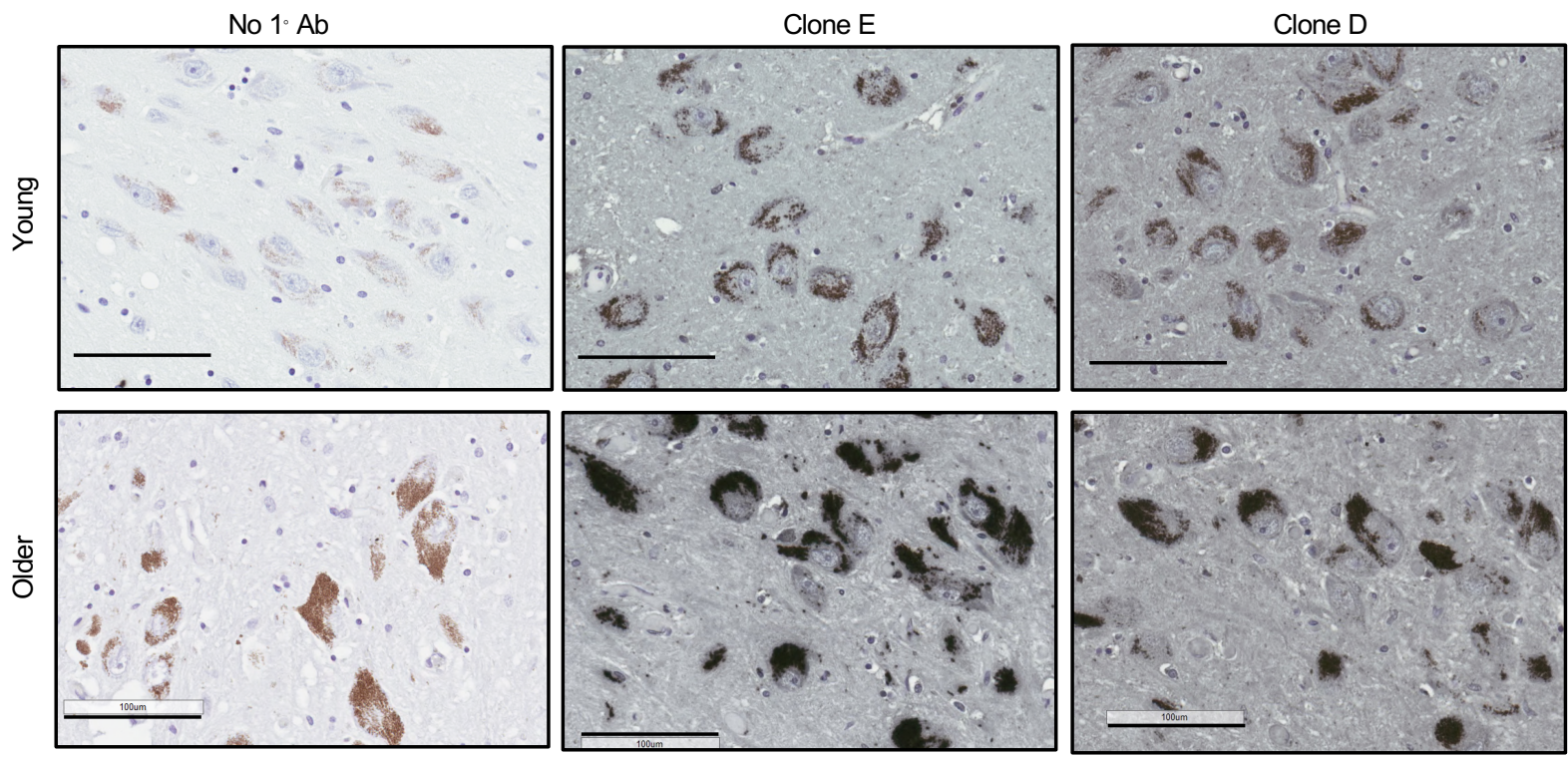

f

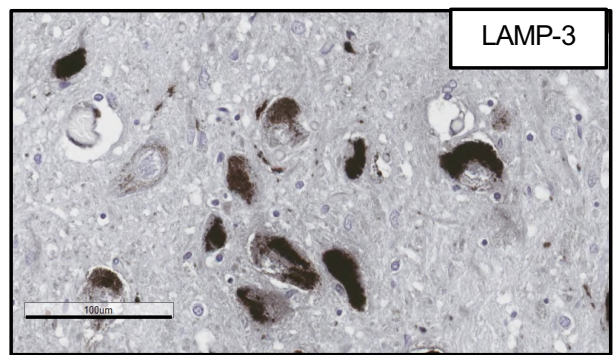

\section{Extended Data Figure 8. Parkin is readily detectable in human brain by routine microscopy}

(a-c) Immunohistochemical detection of parkin in ageing human brain including (a) dopaminergic neurons of the S. nigra, (b) periaqueductal grey neurons in the midbrain and (c) cortex. Parkin was detected using the indicated clones and metalenhanced DAB (black colour).. No primary Ab controls are shown. In all panels, scale bars represent $100 \mu \mathrm{m}$, or as indicated (d-e) Immunohistochemical detection of parkin in S.nigra tissue from from adult control subjects aged (d) 24 and (e) 66 years. Staining using clones E and D respectively and no primary antibody control are shown. Scale bars, $100 \mu \mathrm{m}$.

(f) Immunohistochemical detection of LAMP-3 protein in dopaminergic neurons of the S. nigra from an adult control brain. 


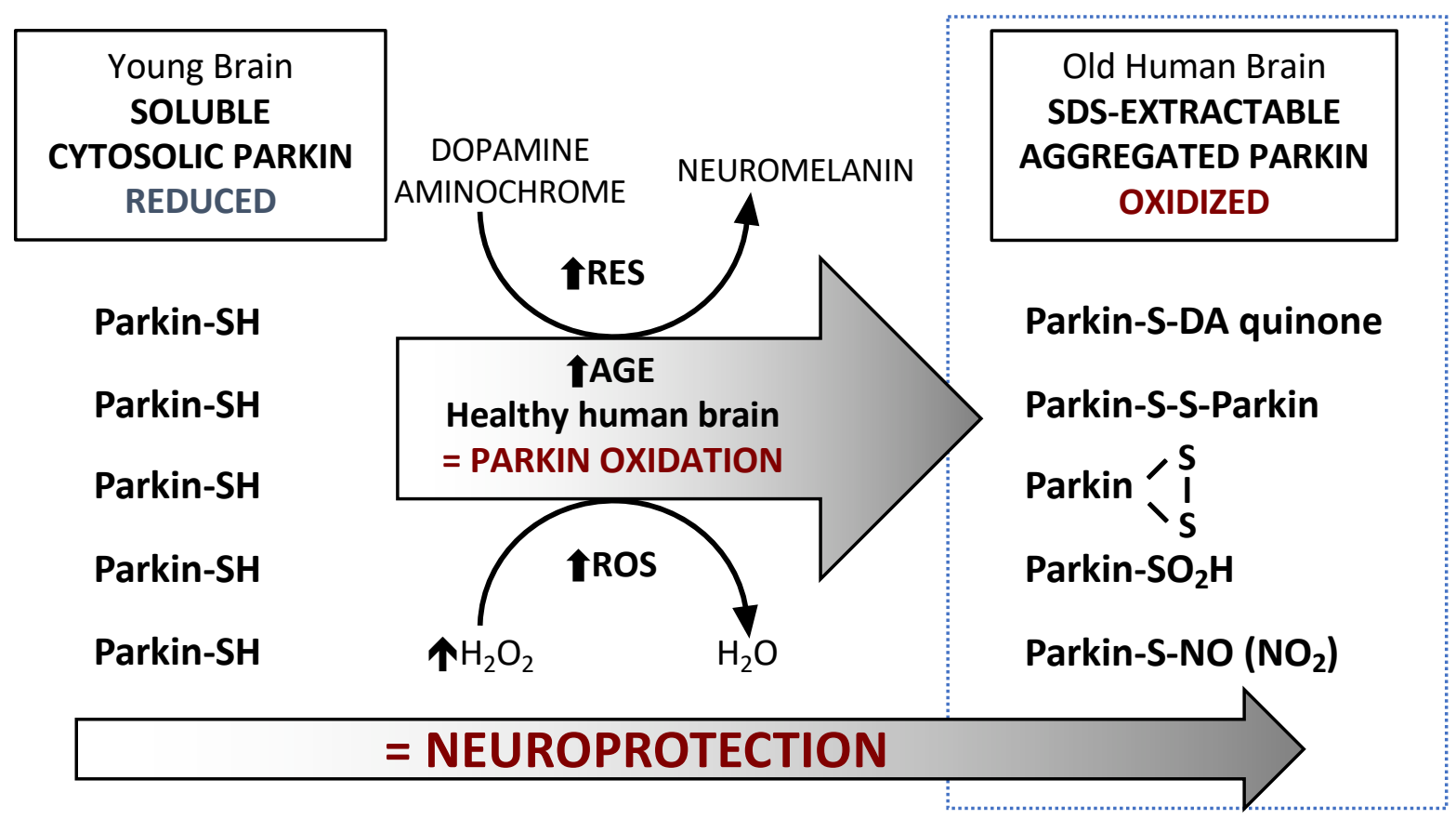

Extended Data Figure 9. Proposed model for parkin's redox functions in human midbrain.

In human brain, parkin thiol (-SH) oxidation neutralizes cellular reactive oxygen species $\left(\mathrm{ROS} ; \mathrm{H}_{2} \mathrm{O}_{2}\right)$ and potentially toxic dopamine (DA) metabolites (DA quinones; RES) during ageing. The oxidation of wild-type parkin promotes higher molecular weight species formation, insolubility and aggregation. During normal ageing, both reversible and irreversible oxidation events occur, which promote parkin's eventual transition into an insoluble, oxidized state by the beginning of the $5^{\text {th }}$ decade. The oxidation and aggregation lead to parkin's accumulation within LAMP-3-positive lysosomes within DA neurons of the $S$. nigra. The multimodal oxidation of parkin confers neuroprotection. In PRKN-linked ARPD, the absence of parkin's redox effects leads to a rise in ROS, reduced sequestration of DA radicals and elevation of RNS. 


\begin{tabular}{|c|c|c|}
\hline & $\begin{array}{c}\text { TS-extractable (IP, } \mathbf{n = 4 )} \\
\text { Mean (range) }\end{array}$ & $\begin{array}{c}\text { SDS-extractable (IP, n=8) } \\
\text { Mean (range) }\end{array}$ \\
\hline$\%$ Parkin protein coverage & $46.8(35-72)$ & $19.4(2-58)$ \\
\hline \# Peptides identified & $15.0(10-23)$ & $6.4(1-17)$ \\
\hline \# Cysteines identified & $17.5(13-26)$ & $6.1(1-16)$ \\
\hline Reduced residues (IAA-Cys) & $17.5(13-26)$ & $6.1(1-16)$ \\
\hline IAA/Total cysteines & $17.5 / 35$ & $6.1 / 35$ \\
\hline$\%$ & 50 & 17.5 \\
\hline IAA/Total identified cysteines & $17.5 / 28$ & $6.1 / 28$ \\
\hline$\%$ & 62.5 & 21.8 \\
\hline IAA/Total cysteines per run & $17.5 / 28$ & $6.1 / 16$ \\
\hline$\%$ & 62.5 & 38.1 \\
\hline Oxidized residues & $\begin{array}{l}\text { Among PTMs preselected } \\
\text { for screening }\end{array}$ & $\begin{array}{c}\text { Among PTMs preselected for } \\
\text { screening }\end{array}$ \\
\hline Cys --> Dha & Cys 95, 196, 238, 241, 253 & Cys 196 \\
\hline NEM-cysteine & Not detected & Cys 253 \\
\hline Sulfinic acid - Cys dioxidation & Not detected & Not detected \\
\hline Sulfonic acid - Cys trioxidation & Cys 95,253 & Not detected \\
\hline Glutathionylation & Not detected & Not detected \\
\hline S-nitrosylation & Not detected & Not detected \\
\hline $\begin{array}{l}\text { Dopamine quinone adduct } \\
\text { formation }\end{array}$ & Not detected & Not detected \\
\hline Methionine oxidation & Met $80,192,327,458$ & Met 80, 192, 327, 458 \\
\hline
\end{tabular}

\section{Extended Data Table 1. Summary of LC-MS/MS results with a focus on thiol redox state of parkin purified from human brain.}

Results for twelve IP runs (TS extracts; $n=4$; SDS extracts, $n=8$ ) of human cortices to identify reduced (IAA-tagged) and reversibly-oxidized (NEM-tagged) thiol residues. Parkin immunoprecipitation (IP) from human brains was performed using magnetic beads conjugated to the Prk8 antibody or a parkin monoclonal antibody (Clone B). Tissue extraction and IP was performed in the presence of IAA. Higher parkin coverage was consistently obtained from the TS-extracted fractions, along with a higher number of IAA-cysteines/total cysteines identified per run, when compared to the SDS-soluble fractions. In addition to IAA and NEM, we preselected for other non-reversible post-translation modifications for screening as indicated. In human brains, we detected irreversibly oxidized cysteines to sulfonic acid (trioxidation), at C95 and C253 and one NEMlabelled and oxidized cysteine, C253. Among the PTMs we preselected for, we also detected cysteine to dehydroalanine (Cys $\rightarrow$ Dha) shift and methionine oxidation. 